\title{
Genetic variation in major phenotypic traits among diverse genetic origins of teak (Tectona grandis L.f.) planted in Taliwas, Sabah, East Malaysia
}

\author{
Gilles Chaix • Olivier Monteuuis • Charles Garcia • \\ David Alloysius • Jikos Gidiman • Roberto Bacilieri • \\ Doreen K. S. Goh
}

Received: 16 October 2010 / Accepted: 7 February 2011 / Published online: 13 July 2011

(C) INRA and Springer Science+Business Media B.V. 2011

\begin{abstract}
- Introduction As a forest species, genetic variability is high in teak (Tectona grandis) and domestication of the species is very recent. The selection effect among qualitative and quantitative traits is therefore expected to be strong. Native provenances and clonal seed orchard families were compared in this study.

- Materials and methods Forty-one genetic origins of teak, including 26 open-pollinated families from a clonal seed orchard in Ivory Coast, were planted in 1997 in a replicated trial at Taliwas, Sabah, East Malaysia.

- Results and discussion The mortality rate and early measurements of height, and diameter at breast height varied substantially between treatments. The largest height $(>18 \mathrm{~m})$ and diameter $(>21 \mathrm{~cm})$ values recorded after
\end{abstract}

104 months were mostly from the clonal seed orchard families, while the lower performances were mainly observed for the native provenances. Narrow sense heritabilities, assessed for the clonal seed orchard families only, increased gradually with age, reaching relatively high values, especially for height at 104 months $\left(h^{2}=0.76\right)$. There were also some highly significant differences between the 41 genetic origins for six qualitative traits observed from 25 to 104 months. Overall, the clonal seed orchard families were marginally less crooked and grew more vertically than the other sources.

- Conclusions These findings provide further evidence of the usefulness of a clonal seed orchard phase in the genetic improvement of teak and the beneficial influence of a wet tropical climate on major economic traits.
Handling Editor: Luc Pâques

G. Chaix $(\bowtie)$

CIRAD, UMR AGAP,

73 rue Jean-François Breton TA A-40/16,

F-34398 Montpellier, Cedex 5, France

e-mail: gilles.chaix@cirad.fr

O. Monteuuis

CIRAD, UMR AGAP,

F-34398 Montpellier, France

C. Garcia

Forestry Division, Yayasan Sabah Group,

Rakyat Berjaya sdn Bhd, 2nd, 4th, 5th \& 6th Floor, UMNO

Building, P.O. Box 60793, 91017 Tawau, Sabah, Malaysia

\author{
D. Alloysius · J. Gidiman \\ Conservation and Environmental Management Division, \\ Yayasan Sabah Group, \\ Rakyat Berjaya sdn Bhd, 5th \& 6th Floor, UMNO Building, \\ P. O. Box 60793, 91017 Tawau, Sabah, Malaysia \\ R. Bacilieri \\ INRA, UMR AGAP 1334, \\ F-34060 Montpellier, France \\ D. K. S. Goh \\ YSG Biotech Sdn Bhd, Yayasan Sabah Group, \\ Voluntary Association Complex, \\ Mile 2 1/2, off Tuaran Road, P.O. Box 11623, 88817 \\ Kota Kinabalu, Sabah, Malaysia
}


Keywords Clonal seed orchard - Genetic improvement . Heritability. Teak

\section{Introduction}

Within the Verbenaceae family, the genus Tectona comprises four species: Tectona grandis L.f., Tectona hamiltoniana Wall, Tectona philippinensis Benth. \& Hook.f., and Tectona abludens formerly identified as a sub-species of $T$. grandis (Kaosa-ard 1999). T. grandis, commonly known as teak, occurs naturally in India, Laos, Thailand, and Myanmar (exBurma) and is considered as sub-spontaneous in Indonesia, where it was introduced some 400-600 years ago (Kaosa-ard 2000; Siswamartana 2000). This large and long-lived tropical tree species is far more popular than the other three species in the genus due to the premium quality of its wood (Kadambi 1972; Keogh 2001; Tewari 1992). This accounts for the extensive establishment of plantations within and beyond its native range, especially since the beginning of the twentieth century (Ball et al. 2000; Pandey and Brown 2000). Malaysia is not an exception to the list, as teak was reported to have been introduced into Pulau Langkawi, Kedah, from Burma in 1915 (Zakaria and Lokmal 1998), and into Sabah, Kota Marudu district, East Malaysia in 1926 by the Dutch Tobacco Company (Lapongan 2000). The increasing worldwide demand for teak wood, and the alarming decline in the currently available resources, has resulted in an intensification of teak planting activities since the early 1970s (Ball et al. 2000; Keogh 2000; Keogh 2001). Choosing the best teak origins remains a crucial requirement to maximise productivity, bearing in mind that teak timber yields and quality are prone to vary considerably depending on planting site conditions (Kaosa-ard 2000; Keogh 2001). Notwithstanding the usefulness of the scientific information that can be drawn from such studies (Kjaer et al. 1995; Madoffe and Maghembe 1988; Pedersen et al. 2007), there is still a lack of knowledge on genetic influences and heritability estimates for traits of major economic values for this species. These arguments prompted us to investigate and compare the performance of different teak origins, including clonal seed orchard (CSO) families established from seeds in 1997 at Taliwas, Sabah, East Malaysia. The corresponding findings constitute the subject matter of the current paper.

\section{Materials and methods}

\subsection{Plant material}

The plant materials were derived from 41 different origins of T. grandis seeds as detailed in Table 1. The terminology used throughout this paper is in accordance with Zobel and Talbert (1984). Uncertainty remained about the genetic relatedness of seeds from seed lots 8367,8668 , and 9999 , and the 26 derived from the clonal seed orchard at La Sangoué, Ivory Coast (Dupuy and Verhaegen 1993), i.e., whether they were collected from one or more trees.

The different seed lots were germinated in sand beds after subjecting the dry fruits to the usual procedure recommended for teak seeds (Tewari 1992). This consisted of alternating overnight soaking and daytime exposure to full sunlight for 7 days. A few days after sowing, young seedlings bearing at least four fully developed leaves and ranging from 2 to $4 \mathrm{~cm}$ in height were potted individually in $10 \times 15 \mathrm{~cm}$ black plastic bags filled with local soil. They were then raised for 3 months under appropriate nursery conditions until they reached an average $15 \mathrm{~cm}$ stem height suitable for field planting. The sample size was determined by the number of plants per origin displaying the same vigour.

2.2 Trial characteristics, analysis criteria, and statistical processing of data

The trial was planted in March 1997 at a place called Taliwas, located at $\mathrm{km} 18$ on the road from Silam to Danum Valley, Sabah, East Malaysia (Lat $4^{\circ} 58^{\prime} \mathrm{N}$, Long $\left.118^{\circ} 13^{\prime} \mathrm{E}\right)$. Monthly temperatures were $26-28^{\circ} \mathrm{C}$ and the mean annual rainfall (MAR) averaged 2,500 $\mathrm{mm}$ without a distinct dry season. The planting area was flat, $40 \mathrm{~m}$ above sea level, at the bottom of a valley, close to a river, and prone to short periods of flooding, which called for the creation of ditches inside and around the trial. The site, whose characteristics are detailed in Table 2, was prepared by ripping and mounding just before planting.

The 41 different plant origins were planted in a randomized complete block design (Williams and Matheson 1994), consisting of three adjacent blocks. Inside each of the three blocks, each origin was represented by an elementary plot of 15 trees planted in three rows of five individuals, each row being separated from the other by a row of buffer plants. The teak trees used as buffers throughout the trial were of similar age and vigour as the experimental material, but from a different genetic source. All the trees were initially planted at a spacing of $4 \mathrm{~m}$ along the row and $2 \mathrm{~m}$ between rows, covering a total area of $29,520 \mathrm{~m}^{2}$. Manual weeding was carried out uniformly throughout the trial at least twice a year. All the buffer trees were felled after 2 years before competition appeared, thereafter increasing the distance between rows from 2 to $4 \mathrm{~m}$, and halving the overall density from 1,250 trees per hectare to 625 trees per hectare. 
Table 1 Background of the 41 teak seed origins that were compared in Taliwas

\begin{tabular}{|c|c|c|c|c|c|c|c|}
\hline $\begin{array}{l}\text { Seed } \\
\text { lot No. }\end{array}$ & Origin & $\begin{array}{l}\text { Genetic composition } \\
\text { of the acquired seeds }\end{array}$ & Longitude & Latitude & $\begin{array}{l}\text { Elevation } \\
\text { (m above } \\
\text { sea level) }\end{array}$ & $\begin{array}{l}\text { Average annual } \\
\text { rainfall } \\
(\mathrm{mm})\end{array}$ & $\begin{array}{l}\text { Average } \\
\text { annual } \\
\text { temperature } \\
\left({ }^{\circ} \mathrm{C}\right)\end{array}$ \\
\hline 8367 & Prov. India, Chandrapur Maharastra & na & $78^{\circ} 46^{\prime} \mathrm{E}$ & na & na & 1,420 & na \\
\hline 8668 & Prov. Thailand Mae Huat, Lampang ${ }^{\mathrm{a}}$ & na & $99^{\circ} 54^{\prime} \mathrm{E}$ & $18^{\circ} 39^{\prime} \mathrm{N}$ & 350 & 900 & 27 \\
\hline 8823 & Prov. India, Sakrebail Karnataka & Mixture of 100 progenies & $75^{\circ} 29^{\prime} \mathrm{E}$ & $13^{\circ} 48^{\prime} \mathrm{N}$ & 600 & 1,000 & 24 \\
\hline 8824 & Prov. India, Vimoli Vir. Karnataka & Mixture of 100 progenies & $74^{\circ} 37^{\prime} \mathrm{E}$ & $15^{\circ} 11^{\prime} \mathrm{N}$ & 600 & 1,500 & 26 \\
\hline 8831 & Prov. India, Karadibetta Karnataka & Mixture of 100 progenies & $75^{\circ} 02^{\prime} \mathrm{E}$ & $14^{\circ} 05^{\prime} \mathrm{N}$ & 650 & 912 & 24 \\
\hline 8832 & Prov. India, Gialegundi Karnataka & Mixture of 100 progenies & $75^{\circ} 17^{\prime} \mathrm{E}$ & $14^{\circ} 05^{\prime} \mathrm{N}$ & 700 & 1,000 & 24 \\
\hline 8833 & Prov. India, Vimoli Vir. Karnataka & Mixture of 100 progenies & $74^{\circ} 37^{\prime} \mathrm{E}$ & $15^{\circ} 11^{\prime} \mathrm{N}$ & 600 & 1,500 & 26 \\
\hline 8835 & Prov. India, Maukal Karnataka & 1 OP family & $76^{\circ} 00^{\prime} \mathrm{E}$ & $12^{\circ} 15^{\prime} \mathrm{N}$ & 850 & 1,532 & 22 \\
\hline 8836 & Prov. India, Maukal Karnataka & 1 OP family & $76^{\circ} 00^{\prime} \mathrm{E}$ & $12^{\circ} 15^{\prime} \mathrm{N}$ & 850 & 1,532 & 22 \\
\hline 8838 & Prov. India, Maukal Karnataka & 1 OP family & $76^{\circ} 00^{\prime} \mathrm{E}$ & $12^{\circ} 15^{\prime} \mathrm{N}$ & 850 & 1,532 & 22 \\
\hline 8839 & Prov. India, Maukal Karnataka & $1 \mathrm{OP}$ family & $76^{\circ} 00^{\prime} \mathrm{E}$ & $12^{\circ} 15^{\prime} \mathrm{N}$ & 850 & 1,532 & 22 \\
\hline 8841 & Prov. India, Maukal Karnataka & 1 OP family & $76^{\circ} 00^{\prime} \mathrm{E}$ & $12^{\circ} 15^{\prime} \mathrm{N}$ & 850 & 1,532 & 22 \\
\hline 8842 & Prov. India, Maukal Karnataka & $1 \mathrm{OP}$ family & $76^{\circ} 00^{\prime} \mathrm{E}$ & $12^{\circ} 15^{\prime} \mathrm{N}$ & 850 & 1,532 & 22 \\
\hline 8844 & Prov. India, Maukal Karnataka & Mixture of 100 progenies & $74^{\circ} 37^{\prime} \mathrm{E}$ & $15^{\circ} 09^{\prime} \mathrm{N}$ & 600 & 1,500 & 26 \\
\hline 9411 & CSO Prov. India Nilambur & 1 OP family & $76^{\circ} 21^{\prime} \mathrm{E}$ & $11^{\circ} 21^{\prime} \mathrm{N}$ & 49 & 2,565 & na \\
\hline 9412 & CSO Ss Tanzania Kihuhwi & 1 OP family & $38^{\circ} 39^{\prime} \mathrm{E}$ & $5^{\circ} 12^{\prime} \mathrm{S}$ & 260 & 1,880 & na \\
\hline 9416 & CSO Ss Ivory Coast Kokondekro & 1 OP family & na & na & na & na & na \\
\hline 9417 & CSO Prov. India Nilambur & 1 OP family & $76^{\circ} 21^{\prime} \mathrm{E}$ & $11^{\circ} 21^{\prime} \mathrm{N}$ & 49 & 2,565 & na \\
\hline 9418 & CSO Prov. India Nilambur & 1 OP family & $76^{\circ} 21^{\prime} \mathrm{E}$ & $11^{\circ} 21^{\prime} \mathrm{N}$ & 49 & 2,565 & na \\
\hline 9426 & CSO Ss Tanzania Mtibwa (Morogoro) & 1 OP family & $37^{\circ} 39^{\prime} \mathrm{E}$ & $6^{\circ} 00^{\prime} \mathrm{S}$ & 460 & 1,160 & na \\
\hline 9429 & CSO Prov. India Nellicutha & 1 OP family & na & na & na & na & na \\
\hline 9430 & CSO Prov. Thailand Mae Huat & 1 OP family & $99^{\circ} 00^{\prime} \mathrm{E}$ & $18^{\circ} 06^{\prime} \mathrm{N}$ & 350 & 1,300 & na \\
\hline 9431 & CSO Ss Tanzania Kihuhwi & 1 OP family & $38^{\circ} 39^{\prime} \mathrm{E}$ & $5^{\circ} 12^{\prime} \mathrm{S}$ & 280 & 1,860 & na \\
\hline 9432 & CSO Prov. Thailand Pong Salee & $1 \mathrm{OP}$ family & $101^{\circ} 01^{\prime} \mathrm{E}$ & $19^{\circ} 08^{\prime} \mathrm{N}$ & 350 & 1,500 & na \\
\hline 9434 & CSO Prov. India Nellicutha & 1 OP family & na & na & na & na & na \\
\hline 9435 & CSO Prov. India Nellicutha & 1 OP family & na & na & na & na & na \\
\hline 9437 & CSO Prov. India Nilambur & 1 OP family & na & na & na & na & na \\
\hline 9439 & CSO Prov. Thailand Huoi-Nam-Oon & 1 OP family & na & na & na & na & na \\
\hline 9440 & CSO Prov. India Nellicutha & 1 OP family & na & na & na & na & na \\
\hline 9442 & CSO Prov. India Nilambur & 1 OP family & na & na & na & na & na \\
\hline 9443 & CSO Prov. India Vernoli Range & 1 OP family & $74^{\circ} 35^{\prime} \mathrm{E}$ & $15^{\circ} 10^{\prime} \mathrm{N}$ & 573 & 2,032 & na \\
\hline 9445 & CSO Prov. India Nellicutha & 1 OP family & na & na & na & na & na \\
\hline 9446 & CSO Prov. India Vernoli Range & 1 OP family & $74^{\circ} 35^{\prime} \mathrm{E}$ & $15^{\circ} 10^{\prime} \mathrm{N}$ & 573 & 2,032 & na \\
\hline 9450 & CSO Prov. India Vernoli Range & 1 OP family & $74^{\circ} 35^{\prime} \mathrm{E}$ & $15^{\circ} 10^{\prime} \mathrm{N}$ & 573 & 2,032 & na \\
\hline 9452 & CSO Prov. India Masale Valley & 1 OP family & $76^{\circ} 10^{\prime} \mathrm{E}$ & $11^{\circ} 55^{\prime} \mathrm{N}$ & 820 & 1,270 & na \\
\hline 9454 & CSO Prov. Laos Paklay & 1 OP family & $106^{\circ} 00^{\prime} \mathrm{E}$ & $15^{\circ} 00^{\prime} \mathrm{N}$ & 120 & 200 & na \\
\hline 9457 & CSO Prov. India Purunakote & 1 OP family & $84^{\circ} 00^{\prime} \mathrm{E}$ & $20^{\circ} 00^{\prime} \mathrm{N}$ & 133 & $1,200-1,500$ & na \\
\hline 9458 & CSO Prov. Thailand Ban Pha Lay & 1 OP family & $99^{\circ} 59^{\prime} \mathrm{E}$ & $18^{\circ} 13^{\prime} \mathrm{N}$ & 200 & 1,100 & na \\
\hline 9459 & CSO Prov. India Masale Valley & 1 OP family & $76^{\circ} 10^{\prime} \mathrm{E}$ & $11^{\circ} 55^{\prime} \mathrm{N}$ & 820 & 1,270 & na \\
\hline 9463 & CSO Ss Ivory Coast Bamoro & 1 OP family & $5^{\circ} 07^{\prime} \mathrm{W}$ & $7^{\circ} 48^{\prime} \mathrm{N}$ & 330 & 1,100 & na \\
\hline 9999 & Ss PNG Ex Brown River & na & $147^{\circ} 14^{\prime} \mathrm{E}$ & $9^{\circ} 20^{\prime} \mathrm{S}$ & 400 & 2,100 & na \\
\hline
\end{tabular}

Prov provenance, in accordance with Zobel and Talbert (1984), $n a$ information not available, $O P$ open pollinated, CSO clonal seed orchard Ivory Coast, Ss seed source, in accordance with Zobel and Talbert (1984)

${ }^{a}$ natural stand 
Table 2 Characteristics of the Taliwas planting site

\begin{tabular}{|c|c|}
\hline Lat. & $4^{\circ} 58^{\prime} \mathrm{N}$ \\
\hline Long. & $118^{\circ} 13^{\prime} \mathrm{E}$ \\
\hline Elevation & $35-45 \mathrm{~m}$ \\
\hline Rainfall regime & $\begin{array}{l}2,500 \mathrm{~mm} \text { MAR withou } \\
\text { a distinct dry season }\end{array}$ \\
\hline Mean monthly temperatures & $26-28^{\circ} \mathrm{C}$ \\
\hline \multicolumn{2}{|l|}{ Soil chemical analyses * } \\
\hline $\mathrm{Na}(\mathrm{me} \%)$ & 0.23 \\
\hline $\mathrm{K}(\mathrm{me} \%)$ & 0.05 \\
\hline $\mathrm{Ca}\left(\mathrm{me}^{0} \%\right)$ & 9.8 \\
\hline $\operatorname{Mg}(\mathrm{me} \%)$ & 7.1 \\
\hline $\mathrm{Fe}(\%)$ & 9.9 \\
\hline Mn (\%) & 0.44 \\
\hline P Total (ppm) & 91.5 \\
\hline P Available (ppm) & 2.1 \\
\hline $\mathrm{Al}(\mathrm{me} \%)$ & 0.02 \\
\hline Org C (\%) & 0.86 \\
\hline $\mathrm{N}(\%)$ & 0.11 \\
\hline $\mathrm{C} / \mathrm{N}$ & 8.8 \\
\hline $\mathrm{pH} \mathrm{H}_{2} \mathrm{O}$ (range of variation) & $6.0-6.3$ \\
\hline CEC & 16.9 \\
\hline \multicolumn{2}{|l|}{ Soil texture ${ }^{a}$} \\
\hline Clay $(\%)$ & 33 \\
\hline Silt $(\%)$ & 19 \\
\hline Fine sand $(\%)$ & 32 \\
\hline Coarse sand (\%) & 16 \\
\hline Soil colour & Greyish \\
\hline Soil classification & Fluvisols \\
\hline
\end{tabular}

${ }^{\text {a }}$ Average values corresponding to 8 soil samples taken at a depth of 0 and $60 \mathrm{~cm}$ and from four different locations representing the whole planted area

\subsection{Analysis criteria and statistical processing of data}

The quantitative data consisted of:

1. Mortality rate "M", established by counting the dead trees out of the total number of trees initially planted.

2. Total tree height " $\mathrm{H}$ " measured with a graduated pole, then with a clinometer when trees became too tall.

3. Diameter at breast height, or "DBH", measured with a tape at about $1.3 \mathrm{~m}$ from the ground.

All these data were recorded for each tree 15, 25, and 104 months after planting.

The mean annual increment in height and in diameter at breast height were deduced from these measurements.

Qualitative data were also obtained for the analyses, assigning scores to the following criteria:

4. Straightness: score of 1 for straight trees, 2 for slightly crooked trees, and 3 for very crooked trees.
5. Forking: score of 1 for trees without any fork, 2 when forked in the upper half of the tree, and 3 when forked in the lower half of the tree.

6. Branching: score of 1 for few lateral and small diameter branches, 2 for medium sized branches, and 3 for a higher frequency of thicker branches.

7. Bending: score of 1 for vertical stems, 2 for stems diverging from 0 to $10^{\circ}$ from the vertical, and 3 for wider angles of stem divergence from the vertical.

8. Fluting: score of 1 for an absence of flutes, 2 for slightly fluted, and 3 for more pronounced flutes.

9. Flowering: score of 1 for absence of flowering, and 2 when bearing flowers or fruits.

In order to minimize bias, these qualitative scores were assigned by one assessor. The statistical analyses were performed using the SAS statistical package, version 9.1 (SAS Institute Inc. 2004), PROC CATMOD and PROC GLM, and more specifically, the SAS CORR procedure for age-age and trait-trait phenotypic correlations. The various traits were analysed using a mixed model, with replicates (three) as a fixed effect and seed "origin $\times$ replicate" interaction and the family as random effects.

The general statistical mixed model applied was:

$\mathrm{Y}_{i j k}=\mu+\mathrm{R}_{i}+\mathrm{O}_{j}+(\mathrm{RO})_{i j}+\epsilon_{i j k}$

Where

$Y_{i j k} \quad$ Observation on the $k$ th individual of the $j$ th seed origin in the $i$ th replicate

$\mu \quad$ Overall mean

$R_{i} \quad$ Fixed effect of the "replicate" factor, $1 \leq i \leq 3$

$O_{j} \quad$ Random effect of the "seed origin" factor, $1 \leq j \leq 41$

$(R O)_{i j} \quad$ Random effect of the interaction between "replicate" and "origin"

$\epsilon_{i j k} \quad$ Residual error

Individual tree heritabilities were calculated for the CSO families only (26 families from 42 seed lots), for which we assumed strict half-sib families using the following formula:

$h^{2}=\frac{4 \widehat{\sigma}_{O}^{2}}{\widehat{\sigma}_{O}^{2}+\widehat{\sigma}_{R^{*} O}^{2}+\widehat{\sigma}_{e}^{2}}$

Where:

$\widehat{\sigma}_{O}^{2} \quad$ is the "origin" variance component

$\widehat{\sigma}_{R^{*} O}^{2}$ is the "origin $\times$ replicate" interaction variance component

$\widehat{\sigma}_{e}^{2} \quad$ is the residual error

The variance components for growth traits were estimated using the restricted maximum likelihood method of the SAS VARCOMP procedure (SAS Institute Inc. 2004). The 
standard error of heritability estimates was calculated following Falconer and Mackey (1996).

\section{Results}

Mortality losses for the whole trial were 2.8\% $(52 / 1,845)$ after 15 months, $10 \%(184 / 1,845)$ after 25 months, and $13 \%(240 / 1,845)$ after 104 months for all seed sources combined. The effect of the various origins on mortality, which was responsible for the variations in sample size, became significant 25 months after planting and was even more pronounced at 104 months, ranging at that time from $2 \%$ for seed lots $8668,9418,9429$, and 9443 to $27 \%$ for seed lots 8836, 9417, 9454, and 9457 (Tables 3 and 4). The age-age correlation of mortality increased as illustrated by the lower mortality correlations found between 4 and 15 months $(r=0.48, p<0.001)$ than between 15 and 25 months $(r=0.71, p<0.0001)$, and finally, between 25 and 104 months $(r=0.95, p<0.0001)$.

The analyses of variance (Table 4 ) indicated that height " $\mathrm{H}$ " and "DBH" were strongly influenced 15, 25, and 104 months after planting by the "replicate" (except for $\mathrm{H}$ after 25 months) and "origin" factors, and by the resulting interaction between those two factors (except for DBH 25 and 104 months after planting). The corresponding mean values are given for each of the 41 origins assessed in Table 3. The best height and DBH scores recorded after 104 months were mostly for the CSO families, while poorer performances were mainly observed for the native provenances. Seed source 9999 (Papua New Guinea ex Brown River) gave similar height and DBH means to those of the CSO families (Table 3, Fig. 1). At 104 months after planting, the lowest mean height value for CSO seed lots was recorded for family 9446 (Prov. India Vernoli Range), which gave rise on average to the shortest trees, whereas family 9443 derived from the same native provenance produced much taller trees (Table 3 ). Interestingly, in addition to performing better for height and DBH after 104 months, the CSO families, together with seed source 9999, appeared to be more homogenous than the natural provenances, as indicated by the relevant $\mathrm{CV}$ values (Table 3). At 104 months after planting, several CSO families from Ivory Coast reached average heights of more than $18 \mathrm{~m}$ (families 9418, 9426, 9430, 9440) after planting, and more than $21 \mathrm{~cm}$ in DBH (family 9430), with some individuals taller than $22 \mathrm{~m}$ (families 9412, 9432; Table 3). Annual height and DBH increments were particularly high in the first 25 months after planting, with mean values of $4.7 \mathrm{~m} /$ year and $4.7 \mathrm{~cm}$,

Table 3 Number of trees recorded (N), relevant mortality (M) and mean values for height and DBH 15, 25 and 104 months after planting for the 41 teak origins - CSO in grey cells - planted at Taliwas

\begin{tabular}{|c|c|c|c|c|c|c|c|c|c|c|c|c|c|c|c|c|c|c|c|c|c|c|c|c|c|c|c|c|c|c|}
\hline \multirow{3}{*}{$\begin{array}{c}\text { Seed } \\
\text { lot } \\
\text { No. }\end{array}$} & \multicolumn{10}{|c|}{ After 15 months } & \multicolumn{10}{|c|}{ After 25 months } & \multicolumn{10}{|c|}{ After 104 months } \\
\hline & \multirow{2}{*}{$\mathbf{N}$} & \multirow{2}{*}{$\begin{array}{l}\mathbf{M} \\
\%\end{array}$} & \multicolumn{4}{|c|}{ Height (m) } & \multicolumn{4}{|c|}{ DBH $(\mathrm{cm})$} & \multirow{2}{*}{$\mathbf{N}$} & \multirow{2}{*}{$\begin{array}{l}\mathbf{M} \\
\%\end{array}$} & \multicolumn{4}{|c|}{ Height (m) } & & & (cm) & & & & & Heigh & t (m) & & & DBH & (cm) & \\
\hline & & & Min & Max & Mean & $\mathrm{CV}$ & Min & Max & Mean & $\mathrm{CV}$ & & & Min & $\operatorname{Max}$ & Mean & $\mathrm{CV}$ & Min & Max & Mean & $\mathrm{CV}$ & & $\%$ & Min & Max & Mean & $\mathrm{CV}$ & Min & Max & Mean & $\mathrm{CV}$ \\
\hline 8367 & 43 & 4 & 4.9 & 9.4 & 7.0 & 16 & 4.1 & 7.6 & 5.8 & 15 & 38 & 16 & 5.0 & 11.5 & 9.1 & 17 & 6.0 & 10.5 & 8.0 & 14 & 37 & 18 & 1.7 & 17.9 & 13.9 & 21 & 2.8 & 24.5 & 13.2 & 24 \\
\hline 8668 & 45 & 0 & 3.4 & 8.7 & 6.8 & 20 & $\begin{array}{l}4.1 \\
3.2\end{array}$ & 8.3 & 6.5 & 18 & 43 & 4 & 3.0 & 12.5 & 8.9 & 25 & 4.0 & 12.5 & $\begin{array}{l}9.0 \\
9.0\end{array}$ & 21 & 44 & 2 & 7.7 & 19.9 & 15.6 & 16 & $\begin{array}{l}2.0 \\
6.4\end{array}$ & 22.8 & 16.5 & 20 \\
\hline 8823 & 44 & 2 & 1.1 & 9.3 & 7.0 & 23 & 0.6 & 8.9 & 6.3 & 27 & 39 & 13 & 4.0 & 12.5 & 10.1 & 17 & 4.9 & 13.1 & 10.0 & 18 & 39 & 13 & 9.9 & 19.7 & 16.3 & 15 & 8.9 & 25.7 & 18.9 & 19 \\
\hline 8824 & 44 & 2 & 4.0 & 9.4 & 6.7 & 22 & 3.5 & 8.6 & 6.3 & 20 & 40 & 11 & 6.5 & 13.5 & 9.8 & 19 & 5.5 & 13.0 & 9.4 & 18 & 39 & 13 & 8.3 & 20.5 & 16.1 & 15 & 8.1 & 25.0 & 17.2 & 21 \\
\hline 8831 & 45 & 0 & 2.3 & 9.0 & 7.0 & 21 & 3.2 & 8.6 & 6.3 & 21 & 37 & 18 & 4.0 & 12.5 & 9.5 & 22 & 6.3 & 13.7 & 9.7 & 17 & 36 & 20 & 9.5 & 20.1 & 15.2 & 17 & $\begin{array}{l}0.1 \\
11.3\end{array}$ & 22.4 & 16.9 & 20 \\
\hline 8832 & 45 & 0 & 2.8 & 9.3 & 6.7 & 28 & 3.8 & 8.3 & 6.1 & 21 & 37 & 18 & 3.5 & 12.5 & 9.9 & 20 & 6.0 & 13.3 & 9.6 & 19 & 37 & 18 & 8.1 & 20.3 & 15.5 & 17 & 8.7 & 22.4 & 16.5 & 21 \\
\hline 8833 & 45 & 0 & 4.5 & 9.9 & $\begin{array}{l}.11 \\
7.3\end{array}$ & 14 & 4.8 & $\begin{array}{l}0.3 \\
7.6\end{array}$ & $\begin{array}{l}.1 \\
6.2\end{array}$ & 11 & 44 & 2 & 4.0 & 12.5 & 9.2 & 22 & 5.5 & 12.0 & 8.7 & 14 & 43 & $\begin{array}{c}10 \\
4\end{array}$ & $\begin{array}{l}.1 \\
7.9\end{array}$ & 21.3 & 15.3 & 17 & $\begin{array}{l}0.1 \\
11.5\end{array}$ & 24.1 & 16.6 & 18 \\
\hline 8835 & 44 & 2 & 2.8 & 9.3 & 6.5 & 23 & 2.2 & 8.6 & 5.9 & 23 & 36 & 20 & 3.0 & 11.5 & 7.7 & 30 & 5.0 & 13.2 & 9.0 & 20 & 35 & 22 & 10.7 & 20.7 & 15.2 & 18 & 9.9 & 23.3 & 15.9 & 22 \\
\hline 8836 & 42 & 7 & $\begin{array}{l}2.0 \\
2.4\end{array}$ & 9.8 & $\begin{array}{l}7.3 \\
7.2\end{array}$ & 24 & $\begin{array}{l}2.2 \\
2.9\end{array}$ & $\begin{array}{l}0.0 \\
9.9\end{array}$ & 6.3 & 23 & 34 & 24 & 5.6 & 14.6 & 9.5 & 22 & $\begin{array}{l}5.0 \\
6.0\end{array}$ & 14.6 & 9.5 & 20 & 33 & 27 & 10.7 & 20.3 & $\begin{array}{l}15.2 \\
15.3\end{array}$ & $\begin{array}{l}10 \\
16\end{array}$ & 10.4 & 24.1 & 15.9 & 20 \\
\hline 8838 & 44 & 2 & 2.7 & 9.0 & 7.0 & 18 & 2.5 & 8.6 & 6.3 & 18 & 42 & 7 & 4.0 & 12.5 & 8.9 & 24 & 6.6 & 13.7 & 9.3 & 17 & 42 & 7 & 6.5 & 20.1 & 15.2 & 21 & 9.3 & 27.2 & 17.6 & 22 \\
\hline 8839 & 44 & 2 & 4.6 & 10.0 & 7.5 & 18 & 3.8 & 9.2 & 6.6 & 17 & 41 & 9 & 2.0 & 13.5 & 8.9 & 32 & 6.2 & 14.0 & 9.7 & 19 & 39 & 13 & 6.9 & 21.5 & 16.1 & 20 & 12.3 & 27.5 & 17.7 & 21 \\
\hline 8841 & 40 & 11 & 1.8 & 8.8 & 6.0 & 26 & 1.9 & 8.0 & 5.5 & 23 & 35 & 22 & 2.5 & 12.0 & 8.1 & 28 & 2.0 & 11.5 & 7.9 & 24 & 32 & 29 & 5.5 & 21.1 & $\begin{array}{l}13.1 \\
13.1\end{array}$ & 21 & 8.8 & 24.6 & 14.9 & 24 \\
\hline 8842 & 44 & 2 & 2.4 & 9.9 & 7.0 & 22 & 2.2 & 9.5 & 6.1 & 22 & 36 & 20 & 3.5 & 13.5 & $\begin{array}{l}8.1 \\
8.9\end{array}$ & 26 & 5.5 & 13.8 & 8.8 & 10 & 24 & 24 & 7.7 & $\begin{array}{l}21.1 \\
20.7\end{array}$ & $\begin{array}{l}15.1 \\
15.6\end{array}$ & 21 & 0.0 & 31.1 & $\begin{array}{l}1.9 \\
16.0\end{array}$ & 30 \\
\hline 8844 & 45 & 0 & 0.8 & 9.2 & 6.6 & 28 & 1.9 & 8.3 & $\begin{array}{l}.1 \\
6.0\end{array}$ & 24 & 42 & 7 & 4.5 & 12.7 & $\begin{array}{l}8.9 \\
8.9\end{array}$ & 24 & 4.5 & $\begin{array}{l}11.8 \\
11.8\end{array}$ & $\begin{array}{l}.0 \\
8.9\end{array}$ & 19 & 40 & 11 & 7.5 & 18.7 & $\begin{array}{l}13.0 \\
13.6\end{array}$ & 20 & 7.0 & 22.2 & 15.2 & 25 \\
\hline 9411 & 44 & 2 & 3.1 & 10.0 & 7.3 & 17 & 3.5 & 9.5 & 7.2 & 15 & 41 & 9 & 4.5 & 12.0 & 9.1 & 18 & 6.0 & 12.5 & 10.2 & 15 & 38 & 16 & 11.9 & 19.9 & 16.9 & 11 & 12.3 & 27.5 & 19.6 & 20 \\
\hline 9412 & 45 & 0 & $\begin{array}{l}3.1 \\
2.6\end{array}$ & 9.7 & 8. & 15 & 3.8 & 9.5 & 7.1 & 14 & $\begin{array}{l}41 \\
40\end{array}$ & 11 & $\begin{array}{l}4.3 \\
5.0\end{array}$ & 14.0 & $\begin{array}{l}9.1 \\
10.8\end{array}$ & $\begin{array}{l}10 \\
16\end{array}$ & $\begin{array}{l}0.0 \\
7.0\end{array}$ & 14. & 10.4 & 15 & 3 & 10 & 11.3 & 22.3 & $\begin{array}{l}18.9 \\
18.0\end{array}$ & 13 & 11.7 & 27.7 & 19.1 & 19 \\
\hline 9416 & 45 & 0 & 4.6 & 10.3 & 7.5 & 18 & 4.5 & 8.6 & 6.6 & 16 & 42 & 7 & 5.5 & 14.5 & 10.4 & 20 & 6.0 & 13.7 & 9.6 & 18 & 40 & 11 & 5.5 & 20.7 & 16.0 & 16 & 7.7 & 25.6 & 17.2 & 20 \\
\hline 9417 & 40 & 11 & 2.0 & 9.0 & 6.8 & 25 & 2.2 & 8.3 & 6.4 & 22 & 36 & 20 & 5.0 & 12.5 & 9.9 & 18 & 5.0 & 13.1 & 9.6 & 18 & 33 & 27 & 12.1 & 20.3 & 15.8 & 13 & 11.9 & 22.6 & 16.8 & 18 \\
\hline 9418 & 45 & 0 & 4.5 & 10.0 & $\begin{array}{l}7.6 \\
7.3\end{array}$ & 19 & 4.1 & $\begin{array}{l}8.9 \\
8.9\end{array}$ & 6.6 & 16 & 44 & 2 & 6.5 & 14.6 & 10.8 & 15 & 7.0 & 13.0 & 9.9 & 15 & 44 & 2 & 14.5 & 21.7 & 18.4 & 9 & 13.2 & 27.1 & 18.7 & 17 \\
\hline 9426 & 43 & 4 & 4.5 & 9.6 & 8. & 15 & 4.5 & 11.1 & 7. & 10 & 72 & 7 & 5 & 13. & 10 & 16 & 5 & 15 & 108 & 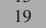 & 4 & 9 & 8. & & & 13 & & & & 19 \\
\hline 9429 & 45 & 0 & 1.4 & $\begin{array}{l}9.0 \\
9.9\end{array}$ & $\begin{array}{l}0.0 \\
7.3\end{array}$ & 23 & 1.3 & 9.5 & 6.9 & $\begin{array}{l}10 \\
23\end{array}$ & 44 & 2 & 4.5 & 13.5 & 10.2 & 21 & $\begin{array}{l}5.0 \\
6.1\end{array}$ & 14.5 & $\begin{array}{l}10.1 \\
10.1\end{array}$ & 19 & 4 & 2 & $\begin{array}{l}0.1 \\
11.7\end{array}$ & 21.3 & $\begin{array}{l}17.0 \\
17.2\end{array}$ & 13 & $\begin{array}{l}.0 .0 \\
9.3\end{array}$ & 25.7 & 18.1 & 19 \\
\hline 9430 & 40 & 11 & 3.2 & 10.7 & 7.6 & 23 & 1.0 & 9.5 & 6.9 & 27 & 37 & 18 & 6.5 & 14.0 & 10.8 & 15 & 7.0 & 14.4 & 10.7 & 18 & 35 & 22 & 12.7 & 21.9 & 18.6 & 9 & 15.7 & 30.6 & 21.1 & 18 \\
\hline 9431 & 43 & 4 & $\begin{array}{l}5.2 \\
3.0\end{array}$ & 9.8 & 0 & 20 & $\begin{array}{l}1.0 \\
3.8\end{array}$ & 9.2 & $\begin{array}{l}0.9 \\
6.8\end{array}$ & 18 & 31 & $\begin{array}{l}10 \\
13\end{array}$ & 0.3 & 14.0 & 10.4 & 24 & 5.5 & $\begin{array}{l}14.4 \\
13.5\end{array}$ & 10.1 & 10 & 33 & 22 & 10.3 & & & 17 & & & 19.3 & $\begin{array}{l}10 \\
20\end{array}$ \\
\hline 9432 & 45 & 0 & 5.2 & 8.9 & 7.2 & 13 & 4.5 & 8.6 & 6.8 & 15 & 43 & 4 & 5.0 & 13.5 & 9.2 & 18 & 6.0 & 12.0 & 9.6 & 14 & 41 & 9 & 11.5 & 22.3 & $\begin{array}{l}17.4 \\
17.1\end{array}$ & 13 & 13.1 & 26.3 & 18.5 & 19 \\
\hline 9434 & 43 & 4 & 2.6 & 9.7 & 7.2 & 21 & 3.2 & 8.9 & 6.9 & 16 & 41 & 9 & 2.5 & 14.5 & 10.0 & 26 & 5.5 & 12.5 & 9.9 & 17 & 41 & 9 & 11.7 & 21.1 & 16.9 & 14 & 11.0 & 25.9 & 18.4 & 19 \\
\hline 9435 & 45 & 0 & 4.6 & 10.6 & 2 & 15 & 4.1 & 8.6 & 6.9 & 15 & $\begin{array}{l}41 \\
43\end{array}$ & 4 & 5.0 & 13.0 & & 14 & 6.0 & 13.0 & (10) & 17 & 4 & 4 & 11.3 & & & 12 & & 24.0 & $\begin{array}{l}18.4 \\
18.6\end{array}$ & 19 \\
\hline 9437 & 44 & 2 & 2.3 & 10.2 & 7. & 22 & 1.9 & 9. & 6.9 & 22 & 40 & 11 & 5. & 15.0 & 11 & 20 & 5. & 14.0 & 10.5 & 18 & 40 & 11 & 13.7 & 21.9 & 17.5 & 11 & 12.8 & 26.3 & 19.4 & 18 \\
\hline 9439 & 45 & 0 & 3.4 & 10.3 & 8. & 16 & 2.5 & 9.5 & 7.4 & 18 & 43 & 4 & 6.5 & 14.0 & 11.0 & 15 & 6.5 & 15.0 & 10.6 & 16 & 41 & 9 & 14.1 & 21.1 & 17.8 & 10 & 12.6 & 26.4 & 18.8 & 18 \\
\hline 9440 & 44 & 2 & 4.6 & 10.3 & 7.6 & 17 & 5.1 & 10.8 & 7.4 & 16 & 41 & 9 & 4.5 & 13.5 & 10. & 19 & 7.0 & 16.5 & 10 & 15 & 4 & 9 & 15.9 & 21.3 & 18 & 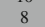 & 14.5 & 25.8 & 19.9 & 14 \\
\hline 9442 & 45 & 0 & 4.9 & 9.0 & 7. & 12 & 4.5 & 9.2 & 7.3 & 14 & 43 & 4 & 5 & 12 & 9. & 17 & 7 & 13 & 10 & 13 & 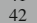 & 7 & 14.1 & 19.9 & 17 & & 13.0 & 25.4 & 19.0 & 16 \\
\hline 9443 & 45 & 0 & 2.6 & 10.7 & 9. & 15 & 2.9 & 9.5 & 7.9 & 1 & 44 & 2 & 7.4 & 16.0 & 11. & 16 & 8.0 & 14 & 11. & 13 & 44 & 2 & 14.5 & 20.7 & 18.0 & 9 & 7.1 & 25.4 & 19.6 & 16 \\
\hline 9445 & 45 & 0 & 4.5 & 9.6 & 7. & 15 & 4.5 & 10.2 & 7.0 & 15 & 42 & 7 & 7.0 & 13 & 10.8 & 14 & 8.2 & 16.2 & 10 & 14 & 42 & 7 & 12.7 & 21.9 & 17.4 & 11 & 11.8 & 27.3 & 19.0 & 16 \\
\hline 9446 & 43 & 4 & 2.8 & 10.1 & 6 & 25 & 2.5 & 9.5 & 6.4 & 26 & 42 & 18 & 3.0 & 14. & $\begin{array}{l}10.0 \\
9.3\end{array}$ & 27 & $\begin{array}{l}0.2 \\
6.1\end{array}$ & 14.9 & 10 & 14 & 42 & 22 & 8.1 & 19.9 & $\begin{array}{l}15.4 \\
15.7\end{array}$ & 15 & 10.7 & 26.6 & 17.6 & 18 \\
\hline 9450 & 44 & 2 & 1.6 & 9. & 7. & 23 & 1. & 8 & 6. & 2 & 43 & 10 & 3 & 14 & 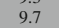 & 2 & 5 & 14 & 10 & 19 & 4 & 22 & 5 & & 17 & 15 & 11.2 & & 18.4 & 19 \\
\hline 9452 & 44 & 2 & 4.1 & 10 & 7. & 22 & 3.8 & 10.8 & 6.7 & 1 & 42 & 7 & 6 & 13 & & 22 & 6.4 & 16 & 10 & 19 & 42 & 7 & 9. & 19.9 & 16.0 & 16 & 10.7 & 24.4 & 17.9 & 19 \\
\hline 9454 & 38 & 16 & 2.3 & 9.8 & 7. & 23 & 2.2 & 9.5 & 6.8 & 22 & 34 & 24 & 3.0 & 13.5 & 9.5 & 19 & 7.0 & 13 & 10.2 & 19 & 33 & 27 & 10.5 & 21.3 & 17.4 & 13 & 12.1 & 26.0 & 19.7 & 15 \\
\hline 9457 & 42 & 7 & 1.7 & 10.0 & 6. & 27 & 2.9 & 9. & 6. & 22 & 38 & 16 & 4 & 12 & 9 & 2 & 5 & 15 & 9. & 2 & 3 & 2 & 10.5 & & 15 & 12 & 10.4 & 29.6 & 17.8 & 24 \\
\hline 9458 & 41 & 9 & 2.3 & 9.4 & 7. & 21 & 2.5 & 8. & 6.7 & 17 & 39 & 13 & 4. & 12 & 9 & 21 & 5 & 14 & 10 & 17 & 3 & 13 & 8. & 20 & 17 & 13 & 7.8 & 29.5 & 19.3 & 20 \\
\hline 9459 & 45 & 0 & 3.9 & 9.6 & 7.5 & 14 & 5.1 & 8.6 & 7.0 & 12 & 42 & 7 & 6.0 & 11.5 & 9.6 & 16 & 7.5 & 14. & 10.3 & 13 & 40 & 11 & 11.9 & 21.5 & 17.7 & 12 & 12.3 & 27.6 & 18.9 & 18 \\
\hline 9463 & 45 & 0 & 4.5 & 9.4 & 7.7 & 11 & 4.8 & $\begin{array}{l}0.0 \\
8.9\end{array}$ & 7.3 & 12 & 44 & 2 & 2.0 & 12.5 & 10.3 & 18 & 7.5 & 13.3 & 10.6 & 15 & 43 & 4 & 14.3 & 20.3 & 17.8 & 8 & 13.6 & 26.2 & 19.6 & 17 \\
\hline 9999 & 45 & 0 & 3.4 & 9.1 & 7.2 & 15 & 2.9 & 9.2 & 6.7 & 15 & 43 & 4 & 4.0 & 13.5 & 10.3 & 21 & 7.5 & 13.0 & 10.7 & 12 & 43 & 4 & 12.9 & 19.9 & 17 & 9 & 12.9 & 24.3 & 19.4 & 15 \\
\hline Mean & 43 & 2.8 & 3.2 & 9.7 & 7.3 & 20 & 3.2 & 9.1 & 6.7 & 19 & 40 & 10 & 4.6 & 13.3 & 9.8 & 21 & 6.1 & 13.7 & 9.9 & 17 & 39 & 13 & 10.2 & 20.7 & 16.5 & 14 & 10.5 & 25.9 & 18.0 & 19 \\
\hline
\end{tabular}

Data are reported as minimum (Min), maximum (Max), mean and coefficient of variation (CV as a\%) values 
Table 4 Significance levels ( $p$ values) of the two "Replicate" and "Seed Origin" experimental factors tested and of their interaction $(\mathrm{R} \times \mathrm{O})$ on the nine traits observed at different dates after planting at Taliwas

\begin{tabular}{|c|c|c|c|c|c|c|c|c|c|c|}
\hline \multirow[t]{2}{*}{ Time (months) } & \multirow[t]{2}{*}{ Factors } & \multicolumn{9}{|l|}{ Traits } \\
\hline & & Mortality & Height & $\mathrm{DBH}$ & Straightness & Forking & Branching & Bending & Fluting & Flowering \\
\hline \multirow[t]{3}{*}{15} & Replicate (R) & $<0.05$ & $<0.0001$ & $<0.0001$ & na & na & na & na & na & na \\
\hline & Origin $(\mathrm{O})$ & NS & $<0.0001$ & $<0.0001$ & na & na & na & na & na & na \\
\hline & $\mathrm{R} \times \mathrm{O}$ & NS & 0.0001 & $<0.05$ & na & na & na & na & na & na \\
\hline \multirow[t]{3}{*}{25} & Replicate (R) & NS & NS & $<0.0001$ & $<0.0001$ & $<0.0001$ & $<0.0001$ & NS & na & na \\
\hline & Origin $(\mathrm{O})$ & $<0.01$ & $<0.0001$ & $<0.0001$ & $<0.0001$ & $<0.05$ & $<0.0001$ & $<0.0001$ & na & na \\
\hline & $\mathrm{R} \times \mathrm{O}$ & NS & $<0.0001$ & NS & $<0.0001$ & $<0.001$ & $<0.0001$ & $<0.05$ & na & na \\
\hline \multirow[t]{3}{*}{104} & Replicate (R) & $<0.05$ & $<0.0001$ & $<0.0001$ & $<0.0001$ & $<0.0001$ & $<0.0001$ & $<0.001$ & NS & NS \\
\hline & Origin $(\mathrm{O})$ & $<0.0001$ & $<0.0001$ & $<0.0001$ & $<0.0001$ & $<0.0001$ & $<0.0001$ & $<0.0001$ & $<0.0001$ & $<0.0001$ \\
\hline & $\mathrm{R} \times \mathrm{O}$ & NS & $<0.0001$ & NS & $<0.0001$ & $<0.05$ & $<0.0001$ & $<0.0001$ & $<0.0001$ & $<0.0001$ \\
\hline
\end{tabular}

and the highest values of $5.6 \mathrm{~m} /$ year and $5.4 \mathrm{~cm} /$ year, respectively, for family 9443; these notably diminished later (Table 5).

The fact that the final values for height in particular, and also for DBH, could barely be predicted from the first assessments made at 15 and 25 months, in that taller trees did not necessarily have a larger $\mathrm{DBH}$, except for CSO family 9430, was consistent with the respective correlation coefficient values summarized in Table 6.

Narrow sense heritabilities for height and DBH increased gradually as the trees aged from 0.35 to 0.76 and from 0.41 to 0.46 for height and $\mathrm{DBH}$, respectively, reaching relatively high values, especially for height at 104 months (Table 7).

The 41 origins assessed had a significant effect on the six qualitative criteria observed 25 and 104 months after planting, notwithstanding replicates and "replicate $\times$ origin" interaction effects (Table 4). The corresponding data are detailed in Table 8. Overall, trees from the CSO families were less crooked and grew more vertically than those from the native provenances (Table 8, Fig. 2). This was particularly obvious for families 9417, 9426, 9437, and 9463, with $100 \%$ of straight trees, and families 9418,9435 , and 9439 with $98 \%$ of straight trees 104 months after planting. In contrast, most of the India Karnataka, Maukal lots, with a special mention for 8835,8836 , and 8838 , were characterized by a strong inclination to bending. The distinction between the CSO families and the other origins was less evident for forking, branching, and fluting (Table 8, Fig. 2). The largest proportion of forkless trees $(32 \%)$ was observed for seed lot 8367 (Provenance India, Maharastra, Chandrapur), followed by the two CSO families 9429 (India Nelicutha) and 9443 (India Vernoli Range) with $20 \%$ and 19\% of forkless trees, respectively. CSO family 9429 (India Nelicutha) and seed lot 8367 (Provenance India, Maharastra, Chandrapur) were also characterized by trees with reduced branching (36\% and $32 \%$ as respective proportions). Another asset of CSO families 9429, 9430, and 9417 was that they were less affected by fluting than the other origins after 104 months, with $86 \%, 83 \%$, and $82 \%$ of fluteless trees,
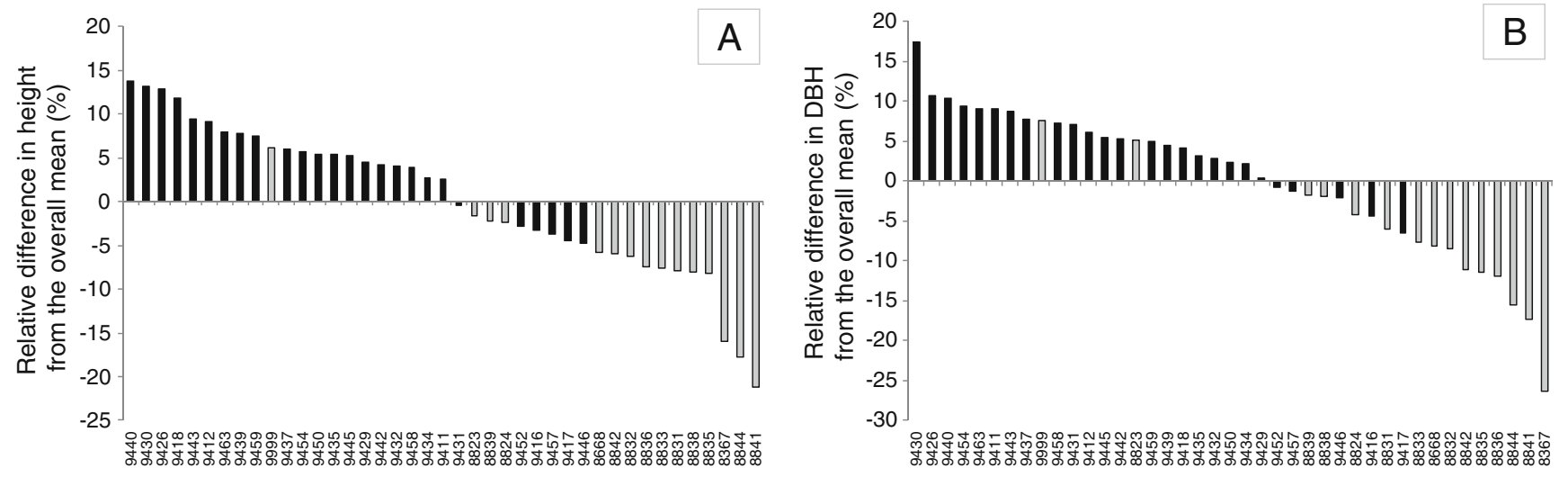

Seed lot

Fig. 1 Relative differences in mean height (a) and in mean DBH (b) for each of the 41 origins - CSO in dark-with the overall average expressed as zero at 104 months 
Table 5 Mean annual increments in height (MAIH, in $\mathrm{m}$ ) and in DBH (MAIDBH, in cm) calculated for the 41 teak origins - CSO in grey cellsplanted at Taliwas at 25 months, between 25 and 104 months and 104 months after planting

\begin{tabular}{|c|c|c|c|c|c|c|c|c|c|c|c|c|c|c|c|c|c|c|c|c|c|c|c|c|}
\hline \multirow[b]{2}{*}{$\begin{array}{c}\text { Seed } \\
\text { lot No. }\end{array}$} & \multicolumn{4}{|c|}{ MAI $\mathrm{H}$ after 25 months } & \multicolumn{4}{|c|}{ MAIDBH after 25 months } & \multicolumn{4}{|c|}{ MAI H 25-104 months } & \multicolumn{4}{|c|}{ MAIDBH 25-104 months } & \multicolumn{4}{|c|}{ MAI H after 104 months } & \multicolumn{4}{|c|}{ MAIDBH after 104 months } \\
\hline & $\min$ & $\max$ & mean & $\begin{array}{l}\text { CV } \\
(\%)\end{array}$ & $\min$ & $\max$ & mean & $\begin{array}{l}\text { CV } \\
(\%)\end{array}$ & $\min$ & $\max$ & mean & $\begin{array}{l}\text { CV } \\
(\%)\end{array}$ & $\min$ & $\max$ & mean & $\begin{array}{l}\text { CV } \\
(\%)\end{array}$ & $\min$ & $\max$ & mean & $\begin{array}{c}\text { CV } \\
(\%)\end{array}$ & $\min$ & $\max$ & mean & $\begin{array}{l}\text { CV } \\
(\%)\end{array}$ \\
\hline 8367 & 2.4 & 5.5 & 4.4 & 17 & 2.9 & 5.0 & 3.8 & 14 & 0.3 & 1.3 & 0.8 & 31 & 0.4 & 2.7 & 0.9 & 45 & 0.3 & 2.8 & 1.5 & 24 & 0.2 & 2.1 & 1.6 & 21 \\
\hline 8668 & 1.4 & 6.0 & 4.3 & 25 & 1.9 & 6.0 & 4.3 & 21 & 0.3 & 1.8 & 1.0 & 31 & 0.1 & 2.1 & 1.2 & 33 & 0.7 & 2.6 & 1.9 & 20 & 0.9 & 2.3 & 1.8 & 16 \\
\hline 8823 & 1.9 & 6.0 & 4.9 & 17 & 2.4 & 6.3 & 4.8 & 18 & 0.1 & 1.6 & 0.9 & 43 & 0.6 & 2.1 & 1.3 & 29 & 1.0 & 3.0 & 2.2 & 19 & 1.1 & 2.3 & 1.9 & 15 \\
\hline 8824 & 3.1 & 6.5 & 4.7 & 19 & 2.6 & 6.2 & 4.5 & 18 & 0.1 & 1.7 & 1.0 & 39 & 0.2 & 2.0 & 1.2 & 37 & 0.9 & 2.9 & 2.0 & 21 & 1.0 & 2.4 & 1.9 & 15 \\
\hline 8831 & 1.9 & 6.0 & 4.6 & 22 & 3.0 & 6.6 & 4.7 & 17 & 0.2 & 2.0 & 0.9 & 45 & 0.4 & 1.8 & 1.1 & 37 & 1.3 & 2.6 & 2.0 & 20 & 1.1 & 2.3 & 1.8 & 17 \\
\hline 8832 & 1.7 & 6.0 & 4.7 & 20 & 2.9 & 6.4 & 4.6 & 19 & 0.3 & 2.1 & 0.9 & 45 & 0.2 & 2.4 & 1.1 & 41 & 1.0 & 2.6 & 1.9 & 21 & 0.9 & 2.3 & 1.8 & 17 \\
\hline 8833 & 1.9 & 6.0 & 4.4 & 22 & 2.6 & 5.8 & 4.2 & 14 & 0.2 & 1.5 & 0.9 & 35 & 0.6 & 2.0 & 1.2 & 29 & 1.3 & 2.8 & 1.9 & 18 & 0.9 & 2.5 & 1.8 & 17 \\
\hline 8835 & 1.4 & 5.5 & 3.7 & 30 & 2.4 & 6.3 & 4.3 & 20 & 0.2 & 1.9 & 1.1 & 38 & 0.2 & 1.9 & 1.1 & 42 & 1.1 & 2.7 & 1.8 & 22 & 1.2 & 2.4 & 1.8 & 18 \\
\hline 8836 & 2.7 & 7.0 & 4.6 & 22 & 2.9 & 7.0 & 4.6 & 20 & 0.3 & 1.8 & 0.9 & 43 & 0.2 & 2.2 & 1.0 & 41 & 1.2 & 2.8 & 1.8 & 20 & 1.2 & 2.3 & 1.8 & 16 \\
\hline 8838 & 1.9 & 6.0 & 4.3 & 24 & 3.2 & 6.6 & 4.4 & 17 & 0.2 & 1.9 & 1.0 & 42 & 0.4 & 2.8 & 1.3 & 38 & 1.1 & 3.1 & 2.0 & 22 & 0.8 & 2.3 & 1.8 & 21 \\
\hline 8839 & 1.0 & 6.5 & 4.2 & 32 & 3.0 & 6.7 & 4.7 & 19 & 0.5 & 1.9 & 1.2 & 30 & 0.6 & 2.6 & 1.2 & 39 & 1.4 & 3.2 & 2.0 & 21 & 0.8 & 2.5 & 1.9 & 20 \\
\hline 8841 & 1.2 & 5.8 & 3.9 & 28 & 1.0 & 5.5 & 3.8 & 24 & 0.1 & 1.6 & 0.8 & 47 & 0.3 & 2.0 & 1.1 & 35 & 1.0 & 2.8 & 1.7 & 24 & 0.6 & 2.4 & 1.5 & 21 \\
\hline 8842 & 1.7 & 6.5 & 4.3 & 26 & 2.6 & 6.6 & 4.2 & 19 & 0.2 & 1.8 & 1.0 & 40 & 0.2 & 3.0 & 1.1 & 47 & 0.7 & 3.6 & 1.8 & 30 & 0.9 & 2.4 & 1.8 & 21 \\
\hline 8844 & 2.2 & 6.1 & 4.3 & 24 & 2.2 & 5.7 & 4.3 & 19 & 0.1 & 1.5 & 0.8 & 43 & 0.2 & 1.7 & 1.0 & 40 & 0.8 & 2.6 & 1.8 & 25 & 0.9 & 2.2 & 1.6 & 20 \\
\hline 9411 & 2.2 & 5.8 & 4.4 & 18 & 2.9 & 6.0 & 4.9 & 15 & 0.4 & 1.8 & 1.2 & 26 & 0.2 & 2.3 & 1.4 & 35 & 1.4 & 3.2 & 2.3 & 20 & 1.4 & 2.3 & 2.0 & 11 \\
\hline 9412 & 2.4 & 6.7 & 5.2 & 16 & 3.4 & 7.1 & 5.0 & 17 & 0.3 & 1.7 & 1.1 & 34 & 0.2 & 2.6 & 1.3 & 38 & 1.4 & 3.2 & 2.2 & 19 & 1.3 & 2.6 & 2.1 & 13 \\
\hline 9416 & 2.6 & 7.0 & 5.0 & 20 & 2.9 & 6.6 & 4.6 & 18 & 0.1 & 1.6 & 0.9 & 36 & 0.5 & 2.3 & 1.2 & 33 & 0.9 & 3.0 & 2.0 & 20 & 0.6 & 2.4 & 1.8 & 16 \\
\hline 9417 & 2.4 & 6.0 & 4.7 & 18 & 2.4 & 6.3 & 4.6 & 18 & 0.3 & 1.8 & 0.9 & 39 & 0.3 & 2.0 & 1.1 & 35 & 1.4 & 2.6 & 1.9 & 18 & 1.4 & 2.3 & 1.8 & 13 \\
\hline 9418 & 3.1 & 7.0 & 5.2 & 15 & 3.4 & 6.2 & 4.8 & 15 & 0.3 & 1.9 & 1.2 & 28 & 0.1 & 2.4 & 1.3 & 33 & 1.5 & 3.1 & 2.2 & 17 & 1.7 & 2.5 & 2.1 & 9 \\
\hline 9426 & 2.4 & 6.5 & 5.2 & 16 & 2.8 & 7.2 & 5.2 & 19 & 0.0 & 1.9 & 1.2 & 28 & 0.3 & 2.1 & 1.4 & 32 & 1.0 & 2.9 & 2.3 & 19 & 1.0 & 2.5 & 2.1 & 13 \\
\hline 9429 & 2.2 & 6.5 & 4.9 & 21 & 2.9 & 7.0 & 4.8 & 19 & 0.5 & 2.0 & 1.1 & 34 & 0.3 & 2.3 & 1.2 & 38 & 1.1 & 3.0 & 2.1 & 19 & 1.4 & 2.5 & 2.0 & 13 \\
\hline 9430 & 3.1 & 6.7 & 5.2 & 15 & 3.4 & 6.9 & 5.2 & 18 & 0.3 & 1.7 & 1.2 & 26 & 0.9 & 3.1 & 1.6 & 30 & 1.8 & 3.5 & 2.4 & 18 & 1.5 & 2.5 & 2.1 & 9 \\
\hline 9431 & 2.4 & 6.7 & 5.0 & 24 & 2.6 & 6.5 & 4.8 & 17 & 0.3 & 2.1 & 0.9 & 44 & 0.7 & 2.9 & 1.5 & 36 & 1.5 & 3.6 & 2.2 & 20 & 1.2 & 2.5 & 1.9 & 17 \\
\hline 9432 & 2.4 & 6.5 & 4.4 & 18 & 2.9 & 5.8 & 4.6 & 14 & 0.6 & 2.0 & 1.2 & 28 & 0.4 & 2.4 & 1.4 & 34 & 1.5 & 3.0 & 2.1 & 19 & 1.3 & 2.6 & 2.0 & 13 \\
\hline 9434 & 1.2 & 7.0 & 4.8 & 26 & 2.6 & 6.0 & 4.8 & 17 & 0.1 & 1.7 & 1.1 & 35 & 0.1 & 2.2 & 1.3 & 32 & 1.3 & 3.0 & 2.1 & 19 & 1.4 & 2.4 & 2.0 & 14 \\
\hline 9435 & 2.4 & 6.2 & 5.1 & 14 & 2.9 & 6.2 & 5.0 & 16 & 0.1 & 2.0 & 1.0 & 34 & 0.1 & 2.2 & 1.2 & 35 & 1.0 & 2.8 & 2.1 & 19 & 1.3 & 2.3 & 2.0 & 12 \\
\hline 9437 & 2.6 & 7.2 & 5.3 & 20 & 2.8 & 6.7 & 5.0 & 18 & 0.3 & 2.4 & 1.0 & 43 & 0.3 & 2.5 & 1.4 & 35 & 1.5 & 3.0 & 2.2 & 18 & 1.6 & 2.5 & 2.0 & 11 \\
\hline 9439 & 3.1 & 6.7 & 5.3 & 15 & 3.1 & 7.2 & 5.1 & 16 & 0.5 & 1.9 & 1.0 & 29 & 0.3 & 2.0 & 1.3 & 33 & 1.5 & 3.0 & 2.2 & 18 & 1.6 & 2.4 & 2.0 & 10 \\
\hline 9440 & 2.2 & 6.5 & 4.9 & 19 & 3.4 & 7.9 & 5.1 & 15 & 0.6 & 2.0 & 1.3 & 29 & 0.8 & 2.1 & 1.4 & 22 & 1.7 & 3.0 & 2.3 & 14 & 1.8 & 2.5 & 2.2 & 8 \\
\hline 9442 & 2.4 & 5.8 & 4.6 & 17 & 3.5 & 6.5 & 5.0 & 13 & 0.6 & 1.9 & 1.1 & 27 & 0.4 & 2.1 & 1.3 & 31 & 1.5 & 2.9 & 2.2 & 16 & 1.6 & 2.3 & 2.0 & 8 \\
\hline 9443 & 3.6 & 7.7 & 5.6 & 16 & 3.8 & 7.0 & 5.4 & 13 & 0.2 & 1.9 & 1.0 & 38 & 0.6 & 2.3 & 1.3 & 29 & 0.8 & 2.9 & 2.3 & 16 & 1.7 & 2.4 & 2.1 & 9 \\
\hline 9445 & 3.4 & 6.7 & 5.2 & 14 & 3.9 & 7.8 & 5.0 & 14 & 0.2 & 1.7 & 1.0 & 30 & 0.4 & 1.9 & 1.3 & 27 & 1.4 & 3.2 & 2.2 & 16 & 1.5 & 2.5 & 2.0 & 11 \\
\hline 9446 & 1.4 & 7.0 & 4.5 & 27 & 2.9 & 7.2 & 4.9 & 21 & 0.5 & 1.6 & 1.0 & 34 & 0.1 & 1.9 & 1.2 & 30 & 1.2 & 3.1 & 2.0 & 18 & 0.9 & 2.3 & 1.8 & 15 \\
\hline 9450 & 1.7 & 7.0 & 4.7 & 21 & 2.6 & 7.1 & 4.8 & 19 & 0.7 & 1.6 & 1.2 & 19 & 0.6 & 2.2 & 1.3 & 28 & 1.3 & 3.0 & 2.1 & 19 & 0.7 & 2.4 & 2.0 & 15 \\
\hline 9452 & 2.9 & 6.5 & 4.6 & 22 & 3.1 & 8.0 & 4.9 & 19 & 0.1 & 1.8 & 1.0 & 44 & 0.1 & 2.3 & 1.2 & 38 & 1.2 & 2.8 & 2.1 & 19 & 1.1 & 2.3 & 1.9 & 16 \\
\hline 9454 & 1.4 & 6.5 & 4.5 & 19 & 3.4 & 6.5 & 4.9 & 19 & 0.7 & 1.7 & 1.2 & 24 & 0.6 & 2.2 & 1.5 & 25 & 1.4 & 3.0 & 2.3 & 15 & 1.2 & 2.5 & 2.0 & 13 \\
\hline 9457 & 1.9 & 6.0 & 4.3 & 26 & 2.7 & 7.5 & 4.8 & 23 & 0.4 & 2.0 & 1.1 & 35 & 0.2 & 2.5 & 1.2 & 41 & 1.2 & 3.4 & 2.1 & 24 & 1.2 & 2.2 & 1.8 & 12 \\
\hline 9458 & 2.2 & 6.0 & 4.5 & 21 & 2.8 & 6.7 & 4.8 & 17 & 0.4 & 1.9 & 1.2 & 31 & 0.3 & 3.0 & 1.4 & 35 & 0.9 & 3.4 & 2.2 & 20 & 1.0 & 2.4 & 2.0 & 13 \\
\hline 9459 & 2.9 & 5.5 & 4.6 & 16 & 3.6 & 6.7 & 5.0 & 13 & 0.6 & 1.9 & 1.2 & 24 & 0.5 & 2.4 & 1.3 & 33 & 1.4 & 3.2 & 2.2 & 18 & 1.4 & 2.5 & 2.0 & 12 \\
\hline 9463 & 1.0 & 6.0 & 5.0 & 18 & 3.6 & 6.4 & 5.1 & 15 & 0.3 & 1.5 & 1.1 & 22 & 0.5 & 2.3 & 1.4 & 30 & 1.6 & 3.0 & 2.3 & 17 & 1.7 & 2.3 & 2.1 & 8 \\
\hline 9999 & 1.9 & 6.5 & 5.0 & 21 & 3.6 & 6.2 & 5.1 & 12 & 0.5 & 2.1 & 1.1 & 27 & 0.1 & 1.9 & 1.3 & 28 & 1.5 & 2.8 & 2.2 & 15 & 1.5 & 2.3 & 2.0 & 9 \\
\hline Mean & 2.2 & 6.4 & 4.7 & 20 & 2.9 & 6.6 & 4.7 & 17 & 0.3 & 1.8 & 1.0 & 34 & 0.4 & 2.3 & 1.2 & 34 & 1.2 & 3.0 & 2.1 & 19 & 1.2 & 2.4 & 1.9 & 14 \\
\hline
\end{tabular}

respectively, versus less than $30 \%$ for several other CSO families (e.g. 19\% for 9431). The natural provenances were generally less prone to fluting than the CSO families (Fig. 2).

Correlations between the various quantitative and qualitative traits analysed indicated weak relationships overall (Table 9), except between straightness and bending 25 ( $r=$ $0.52)$ and $104(r=0.47)$ months after planting, and also at that time, between forking and branching $(r=0.60)$. There were moderately high negative correlations between $\mathrm{H} 25$ and B25 $(r=-0.48)$ and H104 and B104 $(r=-0.33)$.

Surprisingly, for the great majority of the various origins assessed at 104 months, more than $70 \%$ of trees had not entered the flowering stage, except for seed source 9999 (Papua New Guinea ex Brown River) and CSO families

Table 6 Phenotypic correlation coefficients (R) for height $(\mathrm{H})$ and DBH recorded at 15, 25 and 104 months after planting for the combined population of 41 teak origins $(p<0.001)$

\begin{tabular}{lccccc}
\hline Criteria & DBH15 & H25 & DBH25 & H104 & DBH 104 \\
\hline H15 & 0.87 & 0.79 & 0.74 & 0.73 & 0.62 \\
DBH15 & & 0.70 & 0.88 & 0.86 & 0.88 \\
H25 & & & 0.61 & 0.73 & 0.64 \\
DBH25 & & & & 0.88 & 0.90 \\
H104 & & & & & 0.72 \\
\hline
\end{tabular}

9452 and 9459 , with $42 \%, 55 \%$, and $63 \%$ of non-flowering trees, respectively (Fig. 2). At 104 months of age, seed lots $8823,8831,8835,8839,8841$, and 8842 , all originating from India Katamaka natural provenances, stood out from some of the others through a total absence of flowering trees. For 18 other origins, this did not exceed $10 \%$ of trees.

\section{Discussion}

Overall mortality in the trial, averaging $13 \%$ at 104 months, can be considered as low, especially when compared to other teak plantings (Madoffe and Maghembe 1988; Kaosa-ard 2000; Bekker et al. 2004). The high rainfall regime of 2,500 $\mathrm{mm} /$ year, evenly distributed over the year without a distinct dry season, as well as overall good soil

Table 7 Heritabililty (narrow sense), average estimates for height $(\mathrm{H})$, and $\mathrm{DBH}$ recorded at different ages and limited to the $26 \mathrm{CSO}$ families (standard error of estimation in bracket)

\begin{tabular}{lll}
\hline Age (months) & Height & DBH \\
\hline 15 & $0.35( \pm 0.11)$ & $0.41( \pm 0.13)$ \\
25 & $0.55( \pm 0.15)$ & $0.44( \pm 0.13)$ \\
104 & $0.76( \pm 0.19)$ & $0.46( \pm 0.14)$
\end{tabular}


Table 8 Number of trees recorded (N), relevant mortality (M) and mean values 104 months after planting for the six qualitative traits assessed on the 41 teak origins - CSO in grey cells - planted at Taliwas

\begin{tabular}{|c|c|c|c|c|c|c|c|c|c|c|c|c|c|c|c|c|c|c|c|c|c|c|c|c|}
\hline \multirow{2}{*}{$\begin{array}{c}\text { Seed } \\
\text { lot No. }\end{array}$} & \multirow{2}{*}{$\mathbf{N}$} & \multirow{2}{*}{$\begin{array}{l}\mathbf{M} \\
\%\end{array}$} & \multicolumn{4}{|c|}{ Straightness } & \multicolumn{4}{|c|}{ Forking } & \multicolumn{4}{|c|}{ Branching } & \multicolumn{4}{|c|}{ Bending } & \multicolumn{4}{|c|}{ Fluting } & \multicolumn{2}{|c|}{ Flowering } \\
\hline & & & Mean & $\mathrm{C} 1$ & C2 & $\mathrm{C3}$ & Mean & C1 & C2 & $\mathrm{C3}$ & Mean & C1 & $\mathrm{C} 2$ & $\mathrm{C} 3$ & Mean & C1 & $\mathrm{C} 2$ & C3 & Mean & C1 & $\mathrm{C} 2$ & $\mathrm{C} 3$ & Mean & $\mathrm{NF}^{*}$ \\
\hline 8367 & 37 & 18 & 1.1 & 92 & 8 & 0 & 2.1 & 32 & 27 & 41 & 2.0 & 32 & 32 & 36 & 1.4 & 68 & 30 & 2 & 1.4 & 59 & 38 & 3 & 1.1 & 95 \\
\hline 8668 & 44 & 2 & 1.1 & 91 & 9 & 0 & 2.6 & 9 & 23 & 68 & 2.3 & 9 & 52 & 39 & 1.1 & 89 & 11 & 0 & 1.7 & 39 & 50 & 11 & 1.1 & 89 \\
\hline 8823 & 39 & 13 & 1.1 & 92 & 8 & 0 & 2.3 & 18 & 33 & 49 & 2.0 & 18 & 64 & 18 & 1.4 & 67 & 31 & 2 & 1.3 & 77 & 21 & 2 & 1.0 & 100 \\
\hline 8824 & 39 & 13 & 1.2 & 85 & 15 & 0 & 2.6 & 5 & 31 & 64 & 2.5 & 3 & 49 & 48 & 1.4 & 64 & 28 & 8 & 1.5 & 54 & 38 & 8 & 1.1 & 92 \\
\hline 8831 & 36 & 20 & 1.1 & 94 & 6 & 0 & 2.6 & 6 & 31 & 63 & 2.5 & 8 & 36 & 56 & 1.2 & 78 & 22 & 0 & 1.3 & 69 & 28 & 3 & 1.0 & 100 \\
\hline 8832 & 37 & 18 & 1.1 & 86 & 14 & 0 & 2.8 & 0 & 19 & 81 & 2.6 & 0 & 43 & 57 & 1.2 & 84 & 14 & 2 & 1.6 & 51 & 41 & 8 & 1.0 & 97 \\
\hline 8833 & 43 & 4 & 1.1 & 88 & 12 & 0 & 2.4 & 19 & 23 & 58 & 2.2 & 16 & 47 & 37 & 1.4 & 65 & 28 & 7 & 1.4 & 60 & 37 & 3 & 1.1 & 93 \\
\hline 8835 & 35 & 22 & 1.4 & 63 & 37 & 0 & 2.7 & 3 & 26 & 71 & 2.5 & 0 & 51 & 49 & 1.7 & 49 & 34 & 17 & 1.8 & 31 & 54 & 15 & 1.0 & 100 \\
\hline 8836 & 33 & 27 & 1.3 & 70 & 30 & 0 & 2.8 & 3 & 12 & 85 & 2.6 & 0 & 42 & 58 & 1.6 & 52 & 39 & 9 & 1.6 & 45 & 48 & 7 & 1.1 & 91 \\
\hline 8838 & 42 & 7 & 1.1 & 86 & 14 & 0 & 2.4 & 12 & 36 & 52 & 2.3 & 7 & 57 & 36 & 1.6 & 52 & 36 & 12 & 1.6 & 50 & 36 & 14 & 1.0 & 98 \\
\hline 8839 & 39 & 13 & 1.2 & 79 & 21 & 0 & 2.6 & 5 & 26 & 69 & 2.5 & 3 & 44 & 53 & 1.4 & 72 & 15 & 13 & 1.2 & 82 & 15 & 3 & 1.0 & 100 \\
\hline 8841 & 32 & 29 & 1.1 & 91 & 6 & 3 & 2.8 & 0 & 22 & 78 & 2.7 & 3 & 22 & 75 & 1.4 & 75 & 13 & 12 & 1.4 & 63 & 37 & 0 & 1.0 & 100 \\
\hline 8842 & 34 & 24 & 1.1 & 97 & 0 & 3 & 2.4 & 6 & 50 & 44 & 2.3 & 12 & 50 & 38 & 1.2 & 82 & 12 & 6 & 1.3 & 74 & 26 & 0 & 1.0 & 100 \\
\hline 8844 & 40 & 11 & 1.1 & 90 & 10 & 0 & 2.8 & 5 & 10 & 85 & 2.4 & 8 & 42 & 50 & 1.2 & 85 & 13 & 2 & 1.4 & 65 & 30 & 5 & 1.1 & 88 \\
\hline 9411 & 38 & 16 & 1.0 & 97 & 3 & 0 & 2.9 & 0 & 11 & 89 & 2.6 & 3 & 39 & 58 & 1.1 & 92 & 8 & 0 & 1.4 & 66 & 26 & 8 & 1.1 & 95 \\
\hline 9412 & 37 & 18 & 1.1 & 95 & 5 & 0 & 2.6 & 8 & 27 & 65 & 2.4 & 8 & 41 & 51 & 1.1 & 89 & 8 & 3 & 1.8 & 30 & 62 & 8 & 1.2 & 84 \\
\hline 9416 & 40 & 11 & 1.0 & 98 & 2 & 0 & 2.4 & 15 & 30 & 55 & 2.4 & 13 & 40 & 47 & 1.2 & 88 & 8 & 4 & 1.6 & 45 & 50 & 5 & 1.2 & 83 \\
\hline 9417 & 33 & 27 & 1.0 & 100 & 0 & 0 & 2.7 & 3 & 24 & 73 & 2.2 & 18 & 43 & 39 & 1.0 & 97 & 3 & 0 & 1.2 & 82 & 15 & 3 & 1.0 & 97 \\
\hline 9418 & 44 & 2 & 1.1 & 93 & 7 & 0 & 2.5 & 7 & 34 & 59 & 2.3 & 0 & 68 & 32 & 1.0 & 98 & 2 & 0 & 1.3 & 77 & 18 & 5 & 1.0 & 95 \\
\hline 9426 & 41 & 9 & 1.0 & 100 & 0 & 0 & 2.8 & 2 & 15 & 83 & 2.3 & 0 & 73 & 27 & 1.1 & 93 & 7 & 0 & 1.7 & 37 & 54 & 9 & 1.1 & 85 \\
\hline 9429 & 44 & 2 & 1.1 & 91 & 9 & 0 & 2.3 & 20 & 30 & 50 & 1.8 & 36 & 43 & 21 & 1.1 & 93 & 7 & 0 & 1.1 & 86 & 14 & 0 & 1.0 & 95 \\
\hline 9430 & 35 & 22 & 1.1 & 94 & 6 & 0 & 2.3 & 17 & 37 & 46 & 2.1 & 11 & 63 & 26 & 1.1 & 97 & 0 & 3 & 1.2 & 83 & 17 & 0 & 1.1 & 94 \\
\hline 9431 & 37 & 18 & 1.2 & 81 & 19 & 0 & 2.5 & 14 & 24 & 62 & 2.4 & 16 & 24 & 60 & 1.3 & 78 & 16 & 6 & 1.9 & 19 & 76 & 5 & 1.1 & 86 \\
\hline 9432 & 41 & 9 & 1.1 & 90 & 10 & 0 & 2.8 & 5 & 15 & 80 & 2.3 & 2 & 68 & 30 & 1.3 & 71 & 27 & 2 & 1.9 & 27 & 54 & 19 & 1.1 & 90 \\
\hline 9434 & 41 & 9 & 1.1 & 85 & 15 & 0 & 2.8 & 2 & 17 & 81 & 2.5 & 2 & 41 & 57 & 1.1 & 88 & 10 & 2 & 1.3 & 76 & 20 & 4 & 1.1 & 88 \\
\hline 9435 & 43 & 4 & 1.0 & 95 & 5 & 0 & 2.6 & 12 & 21 & 67 & 2.1 & 16 & 53 & 31 & 1.0 & 98 & 2 & 0 & 1.3 & 74 & 26 & 0 & 1.0 & 95 \\
\hline 9437 & 40 & 11 & 1.0 & 100 & 0 & 0 & 2.4 & 15 & 33 & 52 & 2.2 & 15 & 50 & 35 & 1.1 & 95 & 3 & 2 & 1.3 & 70 & 30 & 0 & 1.1 & 88 \\
\hline 9439 & 41 & 9 & 1.0 & 98 & 2 & 0 & 2.3 & 10 & 46 & 44 & 2.2 & 10 & 61 & 29 & 1.0 & 98 & 2 & 0 & 1.4 & 61 & 39 & 0 & 1.1 & 90 \\
\hline 9440 & 41 & 9 & 1.0 & 95 & 5 & 0 & 2.8 & 2 & 17 & 81 & 2.5 & 0 & 54 & 46 & 1.1 & 85 & 15 & 0 & 1.5 & 51 & 49 & 0 & 1.0 & 95 \\
\hline 9442 & 42 & 7 & 1.1 & 93 & 7 & 0 & 2.4 & 12 & 36 & 52 & 2.3 & 12 & 43 & 45 & 1.1 & 88 & 12 & 0 & 1.5 & 55 & 40 & 5 & 1.1 & 86 \\
\hline 9443 & 44 & 2 & 1.1 & 93 & 7 & 0 & 2.4 & 19 & 23 & 58 & 2.0 & 23 & 52 & 25 & 1.1 & 86 & 14 & 0 & 1.7 & 39 & 50 & 11 & 1.1 & 86 \\
\hline 9445 & 42 & 7 & 1.0 & 95 & 5 & 0 & 2.4 & 14 & 31 & 55 & 2.2 & 12 & 52 & 36 & 1.1 & 90 & 5 & 5 & 1.5 & 55 & 43 & 2 & 1.1 & 90 \\
\hline 9446 & 35 & 22 & 1.1 & 91 & 9 & 0 & 2.9 & 3 & 0 & 97 & 2.4 & 14 & 29 & 57 & 1.2 & 86 & 9 & 5 & 1.6 & 46 & 49 & 5 & 1.3 & 74 \\
\hline 9450 & 42 & 7 & 1.0 & 98 & 2 & 0 & 2.7 & 2 & 29 & 69 & 2.5 & 0 & 52 & 48 & 1.0 & 95 & 5 & 0 & 1.4 & 64 & 36 & 0 & 1.0 & 95 \\
\hline 9452 & 42 & 7 & 1.1 & 88 & 12 & 0 & 2.9 & 0 & 10 & 90 & 2.6 & 0 & 38 & 62 & 1.4 & 76 & 12 & 12 & 2.0 & 29 & 45 & 26 & 1.5 & 55 \\
\hline 9454 & 33 & 27 & 1.1 & 91 & 9 & 0 & 2.7 & 6 & 21 & 73 & 2.4 & 12 & 39 & 49 & 1.3 & 82 & 9 & 9 & 1.5 & 48 & 48 & 2 & 1.1 & 88 \\
\hline 9457 & 33 & 27 & 1.0 & 97 & 3 & 0 & 2.7 & 6 & 21 & 73 & 2.3 & 12 & 48 & 40 & 1.1 & 91 & 9 & 0 & 1.4 & 61 & 36 & 3 & 1.2 & 82 \\
\hline 9458 & 39 & 13 & 1.1 & 90 & 10 & 0 & 2.7 & 0 & 28 & 72 & 2.5 & 3 & 44 & 53 & 1.3 & 74 & 21 & 5 & 1.8 & 38 & 46 & 16 & 1.2 & 79 \\
\hline 9459 & 40 & 11 & 1.3 & 72 & 28 & 0 & 2.6 & 8 & 22 & 70 & 2.4 & 5 & 50 & 45 & 1.3 & 80 & 15 & 5 & 2.0 & 25 & 55 & 20 & 1.4 & 63 \\
\hline 9463 & 43 & 4 & 1.0 & 100 & 0 & 0 & 2.9 & 0 & 9 & 91 & 2.6 & 0 & 40 & 60 & 1.0 & 95 & 5 & 0 & 1.9 & 23 & 60 & 17 & 1.1 & 93 \\
\hline 9999 & 43 & 4 & 1.0 & 95 & 5 & 0 & 2.7 & 5 & 16 & 79 & 2.1 & 5 & 77 & 18 & 1.1 & 88 & 9 & 3 & 1.6 & 42 & 53 & 5 & 1.6 & 42 \\
\hline Mean & 39 & 13 & 1.1 & 91 & 9 & 0 & 2.6 & 8 & 24 & 68 & 2.3 & 9 & 48 & 43 & 1.2 & 82 & 14 & 4 & 1.5 & 54 & 39 & 7 & 1.1 & 89 \\
\hline
\end{tabular}

This includes the distribution ratios (as a\%) in the different classes $\mathrm{C} 1, \mathrm{C} 2$ and $\mathrm{C} 3$ established by scoring the five qualitative criteria (see text) except for flowering $(*: \%$ of non flowering trees)

characteristics, may account for the good survival rate and much higher height and DBH values than in other locations (Bekker et al. 2004; Kadambi 1972; Madoffe and Maghembe 1988). The MAR and duration of the dry season are increasingly being acknowledged as having a determining influence on teak growth (Keiding et al. 1986; Keogh 1979). This observation should encourage the planting of suitable teak germplasm under wet conditions, keeping in mind that natural teak provenances include sites with high rainfall ( $>2,500 \mathrm{~mm} /$ year; Keiding et al. 1986). In this respect, our study provides useful information on teak survival, on growth, and on the qualitative traits studied, under high rainfall conditions, at least up to about 9 years after planting. The marked reduction in Mean Annual Increment (MAI) found for growth after 25 months, from 4.7 to $1 \mathrm{~m}$ for height and from 4.7 to $1.2 \mathrm{~cm}$ for $\mathrm{DBH}$, may have been partly due to increased tree-to-tree competition, despite a drastic reduction in the initial density from 1,250 to 625 trees per hectare 24 months after planting, by thinning all the buffer trees. The high water table at the site also probably limited the depth of root system penetration and may also have contributed to this marked decline in $\mathrm{H}$ and DBH growth. At Longuza (Tanzania), a much higher growth rate was also found in the first few years, with a MAI of 2.3 to $2.7 \mathrm{~m}$ at 5 years as opposed to 1.3 to $1.6 \mathrm{~m}$ at 17 years (Madoffe and Maghembe 1988). Another hypothesis is that, after reaching a certain development in height and diameter, teak allocates a greater share of its metabolism to heartwood formation to the detriment of growth in these dimensions. However, this does not seem to be the case as several observations at the same Taliwas site (Goh et al. 2007; Goh and Monteuuis 2009) have indicated that the heartwood formation process actually starts very early, without any detrimental effects on height and diameter increments. This observation was also reported by Bath (2000). Such early heartwood formation constitutes another reason to plant fast-growing teak trees to be harvested at shorter rotations (Chaix et al. 2008; Goh and Monteuuis 2005).

Our results showed that teak growth and qualitative traits are strongly influenced by provenance (broad sense) effects and, in this respect, our observations were consistent with those of several others (Kaosa-ard 2000; Keiding et al. 1986; Madoffe and Maghembe 1988). The fact that the CSO families grew faster and were of better quality overall, with less between-tree variation than the other provenance 

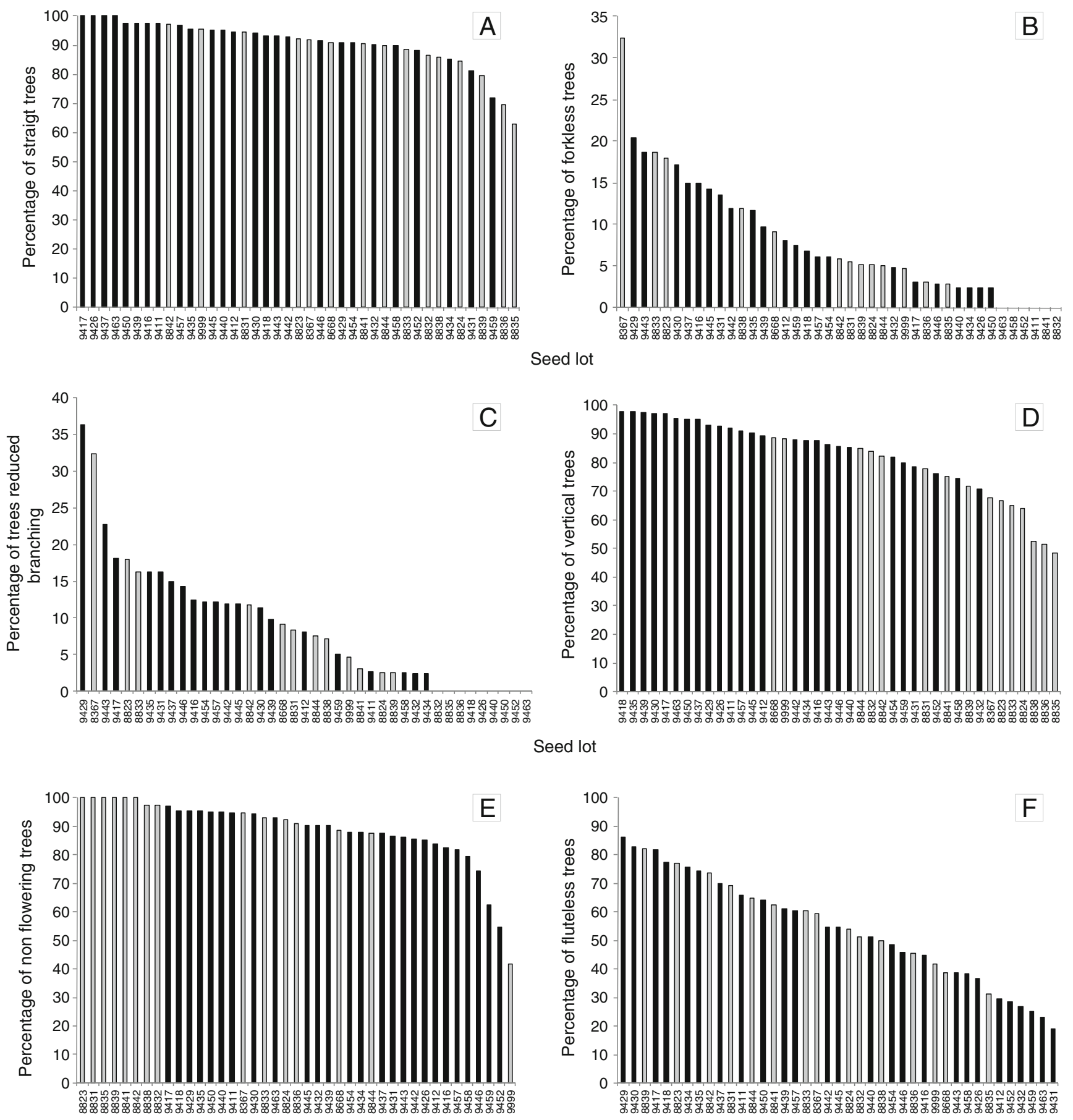

Fig. 2 Proportions (\%) of: a straight trees (scored 1 for straightness), b trees without any fork (scored 1 for forking), c trees with reduced lateral branching (scored 1 for branching), d vertical trees (scored 1

for bending), e trees without any flowers (scored 1 for flowering), $\mathbf{f}$ fluteless trees (scored 1 for fluting) for each origin- CSO in darkassessed 104 months after planting

origins, may have been due to several factors. First of all, these families were derived from superior trees and were each produced by only one mother genotype (Dupuy and Verhaegen 1993), whereas greater uncertainty remained regarding the selection and the number of seed producers from which the provenance and seed source seed lots came. Secondly, the clonal seed orchard at La Sangoué was set up to improve the overall genetic quality of seeds resulting from the intercrossing of superior phenotypes from quite different provenances. Those were soundly selected and 
Table 9 Correlation coefficients for height (H), DBH, straightness (STR), forking (F), bending (B), branching (BR), flowering (FLO) and fluting (FLU) established 25 and 104 months after planting for the 41 teak origins as a whole

\begin{tabular}{|c|c|c|c|c|c|c|c|c|c|c|}
\hline \multirow[t]{2}{*}{ Criteria } & \multicolumn{4}{|c|}{ After 25 months } & \multicolumn{6}{|c|}{ After 104 months } \\
\hline & STR & $\mathrm{F}$ & $\mathrm{B}$ & $\mathrm{BR}$ & STR & $\mathrm{F}$ & $\mathrm{B}$ & FLO & FLU & $\mathrm{BR}$ \\
\hline $\mathrm{H}$ & -0.39 & -0.24 & -0.48 & NS & -0.16 & NS & -0.33 & 0.08 & 0.08 & -0.12 \\
\hline $\mathrm{DBH}$ & -0.20 & NS & -0.23 & 0.14 & -0.13 & 0.11 & -0.20 & 0.15 & 0.18 & NS \\
\hline STR & & 0.34 & 0.52 & NS & & 0.09 & 0.47 & NS & 0.09 & 0.15 \\
\hline $\mathrm{F}$ & & & 0.15 & NS & & & 0.13 & 0.06 & 0.15 & 0.60 \\
\hline B & & & & NS & & & & -0.05 & 0.08 & 0.22 \\
\hline FLO & & & & $\mathrm{Na}$ & & & & & 0.13 & NS \\
\hline FLU & & & & $\mathrm{Na}$ & & & & & & $0.17 *$ \\
\hline
\end{tabular}

$N a$ not available, $N S$ values not indicated as not statistically significant $(p>0.05)$

*significant at $0.001<p \leq 0.05$; other values are statistically significant at $p \leq 0.001$

wisely located within the orchard to favour outcrossing and the capturing of both additive and heterosis genetic effects (Dupuy and Verhaegen 1993), unlike under native stand conditions. The fertilization regime in teak is largely allogamous with high outcrossing rates (Kadambi 1972), as confirmed by genetic marker studies (Indira et al. 2008; Kertadikara and Prat 1995; Kjaer and Suangtho 1995). Hence, different genetic environments and gene flows, depending on the respective physical locations of the grafted genotypes flowering at the same time within the orchard, may have been responsible for the noticeable performance variations found between families derived originally from the same native provenance (CSO families 9446 and 9443 for instance). The conditions, and particularly the climatic characteristics of the sites at which the seeds were produced, can be logically assumed also to play a noticeable role. Between-genotype differences in flowering capacity or synchronism may induce site-related preferential mating systems. Specific combining abilities have also been assumed to play a key role in the genetic quality of seeds produced from CSOs (White et al. 2007; Zobel and Talbert 1984). Some or all of these factors may have been responsible for the better performance of the CSO families compared to the seed lots collected directly from the same original provenances. The comparison between CSO family 9430 and seed lot 8668 from the same native provenance of Mae Huat, Lampang, Thailand, provides a striking example of the great benefit of including a CSO phase in a teak tree improvement and selection programme. Seed source 9999 (Papua New Guinea ex Brown River), assumed to be of seed orchard origin, gave similar mean height and DBH values to those of the CSO families.

For rigour and accuracy reasons, the heritability estimation was limited to the height and DBH quantitative traits of the CSO families, each being the only ones derived from a single mother genotype. The corresponding values found for height and DBH were higher than those reported in the literature (Callister and Collins 2007; Danarto and Hardiyanto 2001). In spite of the fact that the heritability estimation could have been inflated by the single site and by not having strict half-sib families, this suggests that height in teak could be usefully controlled by additive gene actions, contrary to the assumption of Gogate et al. (1997). The slight increment in height and DBH heritability values with age observed here, and for different species (White et al. 2007), might have been due a reduction in environmental variation, which may have been associated with canopy closure and competition for light.

The rationale for estimating genetic correlations between economically important traits, which are currently insufficiently known for teak, to provide information on trait-totrait relationships and for use in tree selection, has already been stressed (Callister and Collins 2007). However, the weakness of the correlation coefficient values obtained failed to provide useful information in this respect, except between straightness and bending, and forking and branching, all of which have major impacts on timber value and the use of teak logs (Baillères and Durand 2000; Ladrach 2009). This is why these traits have been taken into consideration in most teak genetic improvement analyses (Kaosa-ard 1999; Keiding et al. 1986; Kjaer and Lauridsen 1996). The huge benefits of growing teak with reduced lateral branching or natural self-pruning, thereby avoiding the costly and labour-intensive pruning operations practised in a lot of teak plantations to reduce knot and node formations, have to be kept in mind (Bekker et al. 2004; Ladrach 2009).

The first flowering in teak is considered to be an important factor in determining clear bole length, hence tree value. It is initiated in terminal stem buds in almost all trees. The conversion of those buds from the vegetative to 
the reproductive stage disrupts their capacity to ensure bole elongation, while also inducing the formation of two opposite branches, or a fork, as a result of a true dichotomy process. This explains why forking is usually highly and positively correlated with flowering in teak a few years after the first flowers have appeared (Dupuy and Verhaegen 1993; Kaosa-ard 1999). The late onset of flowering in most teak origins in the Taliwas trial, compared to other sites (White 1991) and particularly in Ivory Coast (Dupuy and Verhaegen 1993), where the ortets of the CSO clones were selected, for their delayed flowering among other things, suggests a site effect for flowering onset, as already suggested (Kadambi 1972). The very low correlation $(0.08)$ between height and flowering in our study might have been because only a few trees had entered the flowering stage, and only recently, giving rise to observable forks. There are different types of forks in teak (Kadambi 1972). Most of those recorded in our study differed from flowering-induced forks by the persistence of the main stem which, in the absence of flowering, continued to elongate to reach the great heights recorded. The whorls of large branches at those forks may account for the high positive correlation values found between forking and lateral branching. The surprisingly high proportion of forked trees characterizing the whole trial may be due to the influence of external factors, such as floods or root contact with a high water table, which may have inhibited natural apical dominance, thereby stimulating axillary shoot formation. The fact that most of the forks appeared in the lower half of the trees (Table 8), forming a few years after planting and concomitantly with a growth rate reduction, supports this assumption.

\section{Conclusions}

This study provided useful information on the behaviour of different genetic origins of teak seedling trees as regards the expression of major economic traits. The wet conditions at the site seemed to have a strong positive effect on overall growth performance and also on bole quality. In general, the CSO families traced to selected superior ortets in Ivory Coast, exhibited superiority under the different conditions at Taliwas. This demonstrates the usefulness of CSOs for the genetic improvement of teak, in spite of a few handicaps (Katwal 2008; Kjaer and Foster 1996; Kjaer et al. 2000), and shows that selection in one environment can have positive carry-over effects in another, even when located on a different continent with different climatic and edaphic conditions.

Consistently with other studies (Kjaer et al. 2000), the substantial variation observed between and within the seedling families, even from the same ultimate origins, encourages the use of clones under such favourable wet conditions in order to maximise the early substantial returns in yield and quality of teak plantations. This trial offers huge opportunities for selecting and testing superior genotypes for clonal mass propagation and wise deployment, and also for use as breeding populations for advanced generations of genetic improvement (Goh and Monteuuis 2005).

Acknowledgements The authors are greatly indebted to Dr. Garth Nikles for his numerous useful suggestions and comments on the manuscript of this paper, and Mr. Peter Biggins for improving the English formulation.

\section{References}

Baillères H, Durand PY (2000) Non-destructive techniques for wood quality assessment of plantation-grown teak. Bois et Forêts des Tropiques 263:17-29

Ball JB, Pandey D, Hirai S (2000) Global overview of teak plantations. In: Enters T, Nair CTS (eds) Site, technology and productivity of teak plantations, FORSPA N ${ }^{\circ} 24 / 2000$. Teaknet Publication $N^{\circ}$ 3, Bangkok, pp 11-33

Bath KM (2000) Timber quality of teak from managed plantations of the tropics. Bois et Forêts des Tropiques 263:6-16

Bekker C, Rance W, Monteuuis O (2004) Teak in Tanzania: the Kilombero Valley Teak Co. Ltd. Project. Bois et Forêts des Tropiques 279:11-21

Callister AN, Collins SL (2007) Genetic parameter estimates in a clonally replicated progeny test of teak (Tectona grandis Linn.f.). Tree Genet Genomes 4:237-245

Chaix G, Monteuuis O, Goh DKS, Baillères H, Boutahar N (2008) Quality control and mass production of teak clones for tropical plantations. In: Bhat KM, Balasundaran M, Bhat KV, Muralidharan EM, Thulasidas PK (eds) Proc. of the international symposium held in 2007 on Processing and marketing of Teak wood products of planted forests. Kerala Forest Research Institute, India and International Tropical Timber Organization, Japan, pp 146-157

Danarto S, Hardiyanto EB (2001) Results of the progeny test of teak at 12 years of age at Jember, Esast Java. In: Potential and opportunities in marketing and trade of plantation teak: challenge for the new millenium. Proceeding of the Third Regional Seminar on Teak, Yogyakarta, Indonesia, 31 July-4 August 2000, pp. 249-253.

Dupuy B, Verhaegen D (1993) Le teck de plantation Tectona grandis en Côte d'Ivoire. Bois et Forêts des Tropiques 235:9-24

Falconer DS, Mackay FC (1996) Introduction to quantitative genetics, 4th edn. Longman, London

Gogate MG, Gujar D, Mandal AK, Sharma R, Lal RB, Gupta BN (1997) Genetic analysis of quantitative characters in teak (Tectona grandis). Ann Forest Sci 5:165-167

Goh DKS, Monteuuis O (2005) Rationale for developing intensive teak clonal plantations, with special reference to Sabah. Bois et Forêts des Tropiques 285:5-15

Goh DKS, Monteuuis O (2009) Status of the 'YSG BIOTECH' program of building teak genetic resources in Sabah. Bois et Forêts des Tropiques 301:33-49

Goh DKS, Chaix G, Bailleres H, Monteuuis O (2007) Mass production and quality control of teak clones for tropical plantations: The Yayasan Sabah Group and Forestry Department of Cirad Joint Project as a case study. Bois et Forêts des Tropiques 293:65-77 
Indira EP, Nair PN, Prabba S, Volkaert H (2008) Genetic diversity and contempary gene flow in teak. In: Bhat $\mathrm{KM}$, Balasundaran $\mathrm{M}$, Bhat KV, Muralidharan EM, Thulasidas PK (eds) Proc. of the international symposium held in 2007 on Processing and marketing of Teak wood products of planted forests. Kerala Forest Research Institute, India and International Tropical Timber Organization, Japan, pp 205-213

Kadambi K (1972) Silviculture and management of teak. In: Stephen F (ed) Bulletin 24. AustinState University, Nacogdoches

Kaosa-ard A (1999) Teak (Tectona grandis Linn.f.): Domestication and breeding. Teaknet publication $\mathrm{N}^{\circ} 5 / 1999$.

Kaosa-ard A (2000) Gains from provenance selection. In: Enters T, Nair CTS (eds) Site, technology and productivity of teak plantations, FORSPA N`24/2000. Teaknet Publication No. 3, Bangkok, pp 191207

Katwal RPS (2008) Teak in India: status, prospects and perspectives. In: Bhat KM, Balasundaran M, Bhat KV, Muralidharan EM, Thulasidas PK (eds) Proc. of the international symposium held in 2007 on Processing and marketing of Teak wood products of planted forests. Kerala Forest Research Institute, India and International Tropical Timber Organization, Japan, pp 1-18

Keiding H, Wellendorf H, Lauridsen EB (1986) Evaluation of an international series of teak provenance trials. DANIDA Forest Seed Centre, Humlebaek

Keogh R (1979) Does teak have a future in tropical America. Unasylva 31:13-19

Keogh R (2000) The world of teak plantations. Int Forest Rev 2:123125

Keogh R (2001) New horizons for teak (Tectona grandis Linn. F.) plantations: the consortium support model (CSM) approach of teak 2000. In: Proc. of the Third Regional Seminar on Teak, Potentials and opportunities in marketing and trade of plantation teak: challenge for the new millenium. Yogyakarta, pp. 31-56.

Kertadikara AWS, Prat D (1995) Genetic structure and mating system in teak (Tectona grandis L. f.) provenances. Silvae Genet 44:104 110

Kjaer ED, Foster GS (1996) The economics of tree improvement of Teak (Tectona grandis L.). Technical note no.43. DANIDA Forest Seed Centre, Denmark

Kjaer ED, Lauridsen EB (1996) Results from a second evaluation of DFSC coordinated teak (Tectona grandis) provenance trials: has new information been obtained? In: Proc of Tree Improvement for sustainable tropical forestry, QFRI-IUFRO, Caloundra
Kjaer ED, Suangtho V (1995) Outcrossing rate of teak (Tectona grandis (L.)). Silvae Genet 44:175-177

Kjaer ED, Lauridsen EB, Wellendorf H (1995) Second evaluation of an international series of teak provenance trials. DANIDA Forest Seed Centre, Humlebaek

Kjaer ED, Kaosa-ard A, Suangtho V (2000) Domestication of teak through tree improvement. Options, possible gains and critical factors. In: Enters T, Nair CTS (eds) Site, technology and productivity of teak plantations, FORSPA no. 24/2000. Teaknet Publication No.3, Bangkok, pp 161-189

Ladrach W (2009) Management of teak plantations for solid wood products. ISTF News, Special Report

Lapongan J (2000) Status of teak plantation management in Sabah. In: Proc. of the Seminar on High Value Timber Species for Plantation Establishment-Teak and Mahoganies. 1-2 December 1998, Tawau. Sabah. JIRCAS Working Report No.16, pp. 19-25.

Madoffe SS, Maghembe JA (1988) Performance of teak (Tectona grandis L.f.) provenances seventeen years after planting at Longuza, Tanzania. Silvae Genet 37:175-178

Pandey D, Brown C (2000) Teak: a global overview. FAO/Unasylva 201:3-13

Pedersen AP, Hansen JK, Mtika JM, Msangi TH (2007) Growth, stem quality and age-age correlations in a teak provenance trial in Tanzania. Silvae Genet 56:142-148

SAS (2004) SAS/STAT User's Guide, Cary, NC

Siswamartana S (2000) Productivity of teak plantations in Indonesia. In: Enters T, Nair CTS (eds) Site, technology and productivity of teak plantations, FORSPA No. 24/2000. Teaknet Publication No.3, Bangkok, pp 137-143

Tewari DN (1992) A monograph on teak (Tectona grandis Linn. f.). International book distributors, Dehra Dun, p 479

White KJ (1991) Teak: some aspects of research and develoment. F.A.O. Regional Office for Asia and the Pacific (RAPA), publication 1991/17.

White TL, Adams WT, Neale DB (2007) Forest Genetics. Cabi publishing, Oxfordshire

Williams ER, Matheson AC (1994) Experimental design and analysis for use in tree improvement. CSIRO Victoria

Zakaria I, Lokmal N (1998) Teak in Malaysia. In: Proc. of the 2nd Regional seminar on Teak "Teak for the future", Yangon, Myanmar, FAO-TEAKNET: 199-200.

Zobel B, Talbert J (1984) Applied forest tree improvement. Wiley, New York 